\title{
LA FORMACIÓN DEL ECONOMISTA EN COLOMBIA*
}

\author{
JAIME ANDRÉS SARMIENTO ESPINEL"* \& ADRIANA CAROLINA SILVA ARIAS ${ }^{* * *}$ \\ UNIVERSIDAD MILITAR NUEVA GRANADA
}

Recibido/ Received/ Recebido: 02/12/2013 - Aceptado/ Accepted/ Aprovado: 19/03/2014

\begin{abstract}
Resumen
El objetivo de este artículo es estudiar las características de la educación recibida por los estudiantes de economía en Colombia. Para tal fin, se realizó un análisis de las condiciones socio-económicas, preferencias y desenvolvimiento dentro de la carrera, así como del marco institucional y académico a partir del análisis estadístico de una muestra de 3.404 estudiantes pertenecientes a 60 instituciones de educación superior que ofrecieron el programa de economía en el país durante el año 2007. Se encontró que el 57,04\% eran mujeres, que en promedio tenían 24 años de edad, con padres que alcanzaron niveles educativos de posgrado y secundaria, ingresos por hogar entre 2 y 3 salarios mínimos, que estudiaron en mayor proporción en colegios privados (de calendario A), que sus principales fuentes de financiación fueron recursos familiares y crédito, que el 36,35\% laboraron mientras estudiaron, que dedicaron 87 minutos semanales al estudio independiente por hora de clase y que consultaron tres veces en promedio por semana recursos bibliográficos. Adicionalmente, no se evidenciaron diferencias entre el número de asignaturas y créditos en los planes de estudios de programas acreditados y no acreditados, pero las instituciones acreditadas tuvieron un promedio mayor de monitores, junto a más recursos bibliográficos y físicos.
\end{abstract}

Palabras clave: Calidad en la educación, Desempeño académico, Educación superior, Enseñanza en los programas de economía, Estudiantes de economía, Colombia.

\section{EDUCATION FOR ECONOMIST PROFESSIONALS IN COLOMBIA}

\begin{abstract}
The aim of this paper is to study education characteristics received by economics students in Colombia. To this end, an analysis of socio-economic conditions, preferences and development within the career was developed, as well as an institutional and academic framework from statistical analysis from a sample of 3.404 students considering 60 higher education institutions that offered economics program in the country in 2007. It was found that $57,04 \%$ were women, who were in average 24
\end{abstract}

Artículo de investigación científica y tecnológica, resultado de proyecto de investigación ECO1221: "Formación de economistas en las Instituciones de Educación Superior en Colombia" financiado por la Universidad Militar Nueva Granada. Este producto se enmarca en la línea de investigación en Aprendizaje en Economía del Grupo de Estudios Macroeconómicos - GESMA. Se agradece la colaboración en la ejecución del proyecto de Andrés Virgüez, Sandra Liliana Botón, Diana Carolina Ortiz, Marisol Torres, Gustavo Rueda y Patricia González. Una versión preliminar de este documento fue expuesta en el XIII Encuentro de Decanos, Directores de Programa y Jefes de Departamento de Economía realizado los días 13 y 14 de marzo de 2008 en Santa Marta - Colombia.

** Economista y Magister en Economía de la Universidad Javeriana y Doctor en Economía de El Colegio de México. Profesor investigador de la Facultad de Ciencias Económicas de la Universidad Militar Nueva Granada y Co-líder del Grupo de Estudios Macroeconómicos - GESMA. Correo electrónico: jaime.sarmiento@unimilitar.edu.co

***: Economista de la Universidad del Rosario, Magister en Economía de la Universidad de los Andes y Doctora en Estudios de Población de El Colegio de México. Docente investigadora de la Facultad de Ciencias Económicas de la Universidad Militar Nueva Granada y Co-líder del Grupo de Estudios Macroeconómicos - GESMA. Correo electrónico: adriana.silva@unimilitar.edu.co 
years old, with parents who achieved educational levels and graduate school, household income between 2 and 3 minimum monthly wages, who studied mainly in private schools (calendar A), with main sources of funding such as family funds and loans, $36,35 \%$ of the sample work while they were studying, and spent 87 minutes per week for self-study per hour of class and accessed library resources three times per week on average. Additionally, there is no evidence of differences between classes and number of academic credits in accredited and non-accredited programs curriculum, but accredited institutions had a higher average of monitors, with more bibliographic and physical resources.

Keywords: Quality in education, Academic performance, Higher Education, Training programs in economics, students of economics, Colombia.

\title{
A FORMAÇÃO DO ECONOMISTA NA COLÔMBIA
}

\begin{abstract}
Resumo
O objetivo deste artigo é estudar as características da educação recebida pelos estudantes de economia na Colômbia. Para tal fim, realizou-se uma análise das condiciones socioeconômicas, preferências e desenvolvimento dentro do curso, bem como do marco institucional e acadêmico a partir da análise estatística de uma mostra de 3.404 estudantes pertencentes a 60 instituições de educação superior que ofereceram o programa de economia no país durante o ano 2007. Encontrou-se que os estudantes eram $57,04 \%$ mulheres, tinham em média 24 anos de idade, com pais que atingiram níveis educativos de pós-graduação e secundária, com renda doméstica entre 2 e 3 salários mínimos, estudaram em maior proporção em colégios privados (de calendário A), com suas principais fontes de financiamento em recursos familiares e de créditos, $36,35 \%$ trabalharam enquanto estudavam, dedicaram 87 minutos semanais ao estudo independente por hora de classe e consultaram recursos bibliográficos em média três vezes por semana. Adicionalmente, não se evidenciaram diferenças entre o número de matérias e créditos nos planos de estudos de programas credenciados e não credenciados, mas as instituições credenciadas tiveram uma média maior de monitores, com mais recursos bibliográficos e físicos.
\end{abstract}

Palavras chave: Qualidade na educação, Desempenho acadêmico, Educação superior, Ensino nos programas de economia, Estudantes de economia, Colômbia.

Sarmiento, J. \& Silva, A. (2014) La formación del economista en Colombia. En: Revista de la Facultad de Ciencias Económicas de la Universidad Militar Nueva Granada. rev.fac.cienc.econ, XXII (1).

JEL: A22, I21, I23.

\section{Introducción}

Contar con capital humano calificado es uno de los factores necesarios para el desarrollo y crecimiento económico de un país. Por tanto, la formación profesional a través de la educación superior debe generar y potencializar las competencias de los individuos, permitiendo un mayor bienestar para el individuo y para la sociedad. Además la formación adquirida durante la educación superior tiene mucho que ver con las posibilidades futuras de los estudiantes para insertarse exitosamente al mercado laboral.
Particularmente en el caso de los programas de economía, al igual que en otras disciplinas, en la formación del estudiante entran a interactuar un cúmulo de factores tales como sus características socioeconómicas, sus preferencias y su desenvolvimiento dentro de la carrera, así como el marco institucional y académico en el cual desarrollan sus capacidades. Es por tanto, que un análisis de estos elementos permitiría evidenciar qué tipo de economistas se están formando en Colombia, y de esta manera, poder reflexionar sobre su proyección en la sociedad y el papel fundamental que desempenan los programas 
de economía y las universidades en la calidad de la educación superior.

El objetivo de este artículo es estudiar las características de la educación recibida por los estudiantes de economía en Colombia. Para tal fin, se realizó un análisis de las condiciones socio-económicas, preferencias y desenvolvimiento dentro de la carrera, así como del marco institucional y académico de una muestra de estudiantes de economía durante el 2007.

En la literatura internacional son pocos los estudios de este tipo. Se destacan los de Colander \& Klamer (1987), Colander (2003) y Colander \& Ñopo (2011) los cuales caracterizan el perfil de los estudiantes destacados de posgrado en economía para estudios de caso de Estados Unidos y Chile. Se encuentra además el trabajo realizado por Larroulet \& Domper (2006) sobre los perfiles de alumnos y docentes en las principales universidades de Chile.

La investigación sobre la formación del economista en América Latina fue impulsada por el Banco Interamericano de Desarrollo (BID), a partir de una convocatoria que reunió trabajos de diferentes países de la región. Es el caso de los estudios de Ahumada \& Butler (2009) para México; Espinoza, Machicado \& Makhlouf (2009) para Bolivia y Chile; Rozenwurcel, Bezchinsky \& Rodríguez (2009) para Argentina; los cuales son analizados junto con un borrador de este documento en Lora \& Nopo (2009).

Para el caso colombiano, uno de los primeros estudios en torno a la enseñanza de la economía fue el realizado por la Universidad de Antioquia (2003) el cual se refiere a las metodologías de enseñanza de los docentes de tiempo completo de tres universidades de Colombia, así como a la efectividad e importancia de los mismos en la formación de los estudiantes.

En general, en la literatura los estudios han analizado como máximo seis instituciones, por lo que se debe tener cuidado con la interpretación de los resultados debido a los problemas de representatividad y aleatoriedad. El principal aporte a la literatura que realiza este trabajo tiene que ver con la superación de los inconvenientes sobre el tamaño de la mues- tra, pues se contó con información proporcionada directamente por 32 instituciones, así como con información de otras instituciones recopilada mediante fuentes de información adicionales; logrando compilar una base de datos de 60 instituciones con algún tipo de información, las cuales se encuentran ubicadas en diferentes regiones del país y representan una muestra total de 3.404 estudiantes, quienes se encontraban en mayor proporción $(73,75 \%$ de la muestra nacional) cursando últimos semestres durante el 2007.

Este documento se organiza como sigue. En la segunda sección se presentan los antecedentes de la formación de economistas en Colombia. En la tercera se presentan algunas consideraciones metodológicas acerca del levantamiento de la información. En la siguiente sección se realizará la caracterización de la formación del economista en Colombia, primero de las instituciones y seguidamente de los estudiantes. En la quinta sección se estudiará si existieron perfiles diferenciados de los estudiantes de acuerdo a distintas características de los departamentos, facultades y programas de economía en Colombia. Finalmente, se presentan algunas consideraciones finales.

\section{Antecedentes de la formación de economistas en Colombia}

El origen de la formación de economistas en Colombia está ligado a la disciplina del derecho, dado que en sus inicios se ofrecía como una combinación de ambos tipos de estudios. Entre los antecedentes se encuentra la creación de la Facultad de Ciencias Económicas y Jurídicas de la Universidad Javeriana en 1931 y los estudios no profesionales del Instituto de Economía y Comercio de la Universidad Pontificia Bolivariana en 1943 (Rhenals, 2003).

La enseñanza formal de la economía en Colombia comenzó en la década de los cuarenta. Particularmente, la formación de economistas en instituciones de educación superior públicas se inició a partir de la fundación del Instituto de Ciencias Económicas de la Facultad de Derecho de la Universidad Nacional en 1945, que posteriormente en 1951 se transformó en la Facultad de Economía. A la par, en la Universidad de Antioquia también se originó la Escuela de Cien- 
cias Económicas a partir de la Facultad de Derecho, la cual empezó a funcionar en 1945 (Kalmanovitz, 1993). En el caso de las Universidades privadas, la primera facultad que se fundó fue la de la Universidad de los Andes en 1948, fruto del intento en 1943 de crear una carrera a partir de la combinación de ingeniería industrial y administración de empresas en la Escuela de Administración Industrial y Comercial del Gimnasio Moderno (Kalmanovitz, 1993; Rhenals, 2003).

Actualmente, la mayoría de las instituciones que imparten economía en Colombia fundaron sus programas de economía antes de 1990. Asimismo, esta profesión fue reglamentada a partir de 1969 con la Ley 41; en la cual se establecieron los requisitos para ejercerla, así como la creación del Consejo Nacional Profesional de Economía. Cambios a esta reglamentación se dieron a comienzos de los noventa con la Ley 37 de 1990 y el decreto reglamentario 2890 de 1991.

Desde que se empezó a enseñar de manera formal la economía en el país, el número de profesionales tuvo un crecimiento importante desde mediados de los setenta; sin embargo, el número de graduados ha presentado dos tendencias decrecientes para los períodos 1988-1993 y 2002-2007 (Ilustración 1).

Ilustración 1. Número de economistas graduados 1946$2007^{1}$

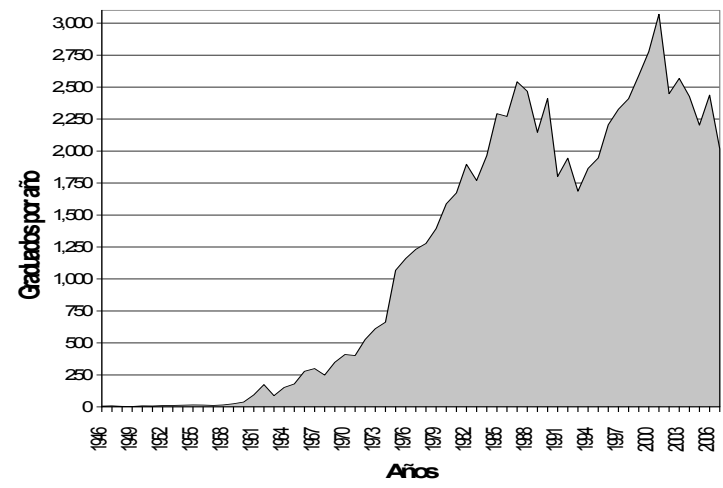

Analizando esta información por departamento, empezaron a aparecer graduados desde este milenio en las ciudades de Barrancabermeja, Montería, Pamplona, Sincelejo, Valledupar y Villavicencio. Asimismo, comparando los años 2000 a 2007 se presentaron caídas de graduados superiores al $50 \%$ para Armenia, Barranquilla, Manizales, Pereira y especialmente Santa Marta; caso contrario en Ibagué y Tunja (Tabla 1).

Uno de los aspectos mencionados dentro de la literatura que podrían incidir en la calidad del perfil de los estudiantes es la diversificación y proliferación de otras carreras afines a la economía, las cuales entran a competir por los candidatos con mayores dotaciones iniciales e incidiendo en el número final de profesionales de esta disciplina. Así, se encontró que el número de solicitudes de inscripción para los programas de economía y carreras afines aumentaron durante el período 2000-I a 2006-I en $20,92 \%$ y $22,29 \%$ respectivamente. Sin embargo, el número de matriculados ha disminuido para economía $(12,06 \%)$ y las carreras comúnmente asociadas a ésta como Administración y Contaduría Pública $(4,81 \%$ y $7,52 \%$, respectivamente) y ha aumentado para otras no tan afines a la economía como Ciencia Política-Relaciones Internacionales, Ingeniería Industrial y Matemáticas-Estadística $(165,02 \%, 9,37 \%$ y $29,61 \%$, respectivamente). La Tabla 2 presenta el comportamiento del número de inscritos, matriculados y estudiantes de primer semestre en economía y carreras afines.

Ante tal panorama de disminución en la demanda de formación en economía y debido a la búsqueda del mejoramiento continuo en la calidad de la educación superior y la formación de profesionales que cumplan con los requerimientos de la sociedad, surge la necesidad de realizar una caracterización de los estudiantes y las instituciones que ofrecieron los diferentes programas de economía en Colombia.

1 Fuente: Consejo Nacional Profesional de Economía (2008). Cálculos de los autores. 
Tabla 1. Número de economistas graduados por departamento 2000-2007²

\begin{tabular}{|c|c|c|c|c|c|c|c|c|}
\hline \multirow{2}{*}{ Ciudad } & \multicolumn{8}{|c|}{ Graduados } \\
\hline & 2000 & 2001 & 2002 & 2003 & 2004 & 2005 & 2006 & 2007 \\
\hline Armenia & 104 & 54 & 37 & 51 & 27 & 14 & 50 & 29 \\
\hline Barrancabermeja & 0 & 0 & 0 & 0 & 0 & 2 & 2 & 1 \\
\hline Barranquilla & 124 & 136 & 74 & 124 & 93 & 86 & 101 & 35 \\
\hline Bogotá & 1.327 & 1.533 & 1.163 & 1.311 & 1.206 & 970 & 1.104 & 962 \\
\hline Bucaramanga & 115 & 149 & 169 & 58 & 113 & 90 & 115 & 84 \\
\hline Cali & 290 & 314 & 223 & 263 & 253 & 280 & 287 & 286 \\
\hline Cartagena & 86 & 82 & 49 & 60 & 83 & 76 & 60 & 66 \\
\hline Cúcuta & 0 & 19 & 56 & 1 & 0 & 0 & 0 & 0 \\
\hline Exterior & 5 & 6 & 1 & 5 & 3 & 0 & 0 & 0 \\
\hline Ibagué & 3 & 32 & 46 & 66 & 69 & 52 & 73 & 21 \\
\hline Manizales & 247 & 193 & 132 & 103 & 84 & 87 & 78 & 65 \\
\hline Medellín & 215 & 222 & 221 & 216 & 208 & 192 & 201 & 151 \\
\hline Montería & 0 & 0 & 0 & 0 & 1 & 44 & 26 & 13 \\
\hline Pamplona & 0 & 0 & 0 & 0 & 0 & 0 & 10 & 10 \\
\hline Pasto & 45 & 29 & 36 & 25 & 50 & 51 & 31 & 48 \\
\hline Pereira & 137 & 150 & 114 & 160 & 54 & 31 & 34 & 31 \\
\hline Popayán & 15 & 72 & 31 & 10 & 15 & 11 & 22 & 10 \\
\hline Santa Marta & 26 & 22 & 32 & 19 & 24 & 25 & 80 & 0 \\
\hline Sincelejo & 0 & 0 & 1 & 1 & 11 & 56 & 5 & 11 \\
\hline Valledupar & 0 & 0 & 0 & 0 & 49 & 20 & 20 & 20 \\
\hline Villavicencio & 0 & 0 & 0 & 0 & 0 & 37 & 64 & 63 \\
\hline Total & 2.777 & 3.069 & 2.448 & 2.568 & 2.427 & 2.202 & 2.438 & 2.017 \\
\hline
\end{tabular}

\section{Algunas consideraciones metodológicas acerca del levantamiento de la información}

La información acerca de las características de las instituciones de educación superior en Colombia que ofrecieron un programa de economía durante el 2007 se levantó directamente con las instituciones y a partir de fuentes secundarias como el Ministerio de Educación, el ICFES y la información disponible en la web. De esta forma, se cuenta con información proporcionada directamente por 32 instituciones, así como la recopilada mediante otras fuentes de información, para finalmente contar con una base de datos de 60 instituciones con algún tipo de información, las cuales se encontraban ubicadas en diferentes regiones del país ${ }^{3}$.

Para realizar la caracterización de los programas y universidades, se envió a las instituciones un cuestionario donde se indagaba por los recursos académicos, administrativos y físicos con los que contaron los estudiantes. De los aspectos curriculares, se analizaron los planes de estudios por créditos y por áreas ${ }^{4}$.

Fuente: Consejo Nacional Profesional de Economía (2008). Cálculos de los autores.

No se especifican los nombres de las instituciones debido a que en el proceso del levantamiento de la información garantizamos a los sujetos de investigación la anonimización de la información.

4 La clasificación de las asignaturas fue realizada por economistas con base al título de la asignatura. Cuando no se consideró que el título expresaba el contenido de la asignatura, se buscaron otras herramientas para clasificarlas, como buscar el programa por la web. 
Tabla 2. Número de Inscritos, Matriculados y Estudiantes de primer semestre según Núcleo Básico de Conocimiento $2000-$ I a $2006-\left.\right|^{5}$

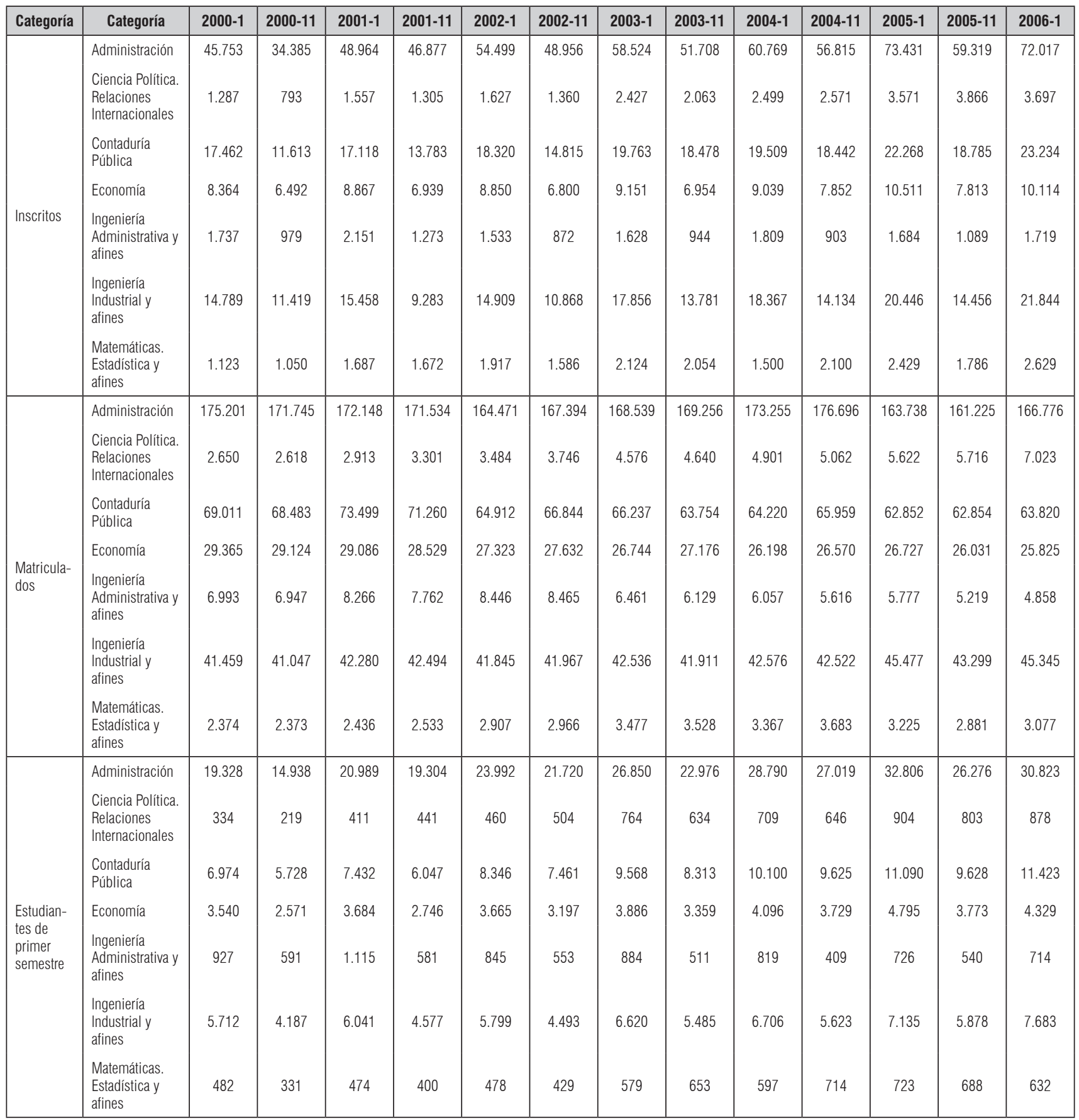

5 Fuente: Sistema Nacional de Información de Educación Superior -SNIES, Ministerio de Educación Nacional (2008). Cálculos de los autores. 
Las áreas fueron definidas tal como lo plantea la Resolución 2774 de 2003 del Ministerio de Educación Nacional6; dichas áreas son la básica ${ }^{7}$, la profesional $^{8}$ y la socio-humanística ${ }^{9}$. No se consideraron en esta clasificación las Prácticas y/o Trabajos de Grado obligatorios, Electivas, las asignaturas de la temática Ambiental- Ecología, entre otras asignaturas; al no poseer la información suficiente para distinguir si su finalidad es de la formación en el área básica, instrumental, énfasis o socio-humanística.

También se solicitó información de los recursos administrativos necesarios para la gestión de la formación integral del economista. Además, se preguntó por el valor de la matrícula, si los estudiantes contaron con monitores, el estatus de acreditación de la calidad del programa de economía y de las instituciones, así como los recursos bibliográficos y físicos con los que contaron los estudiantes.

Por otro lado, para realizar la caracterización estudiantil, aplicamos encuestas a los alumnos que estaban cursando estudios de economía durante el año 2007, así como se complementó con la información del formato de inscripción al Examen de Calidad de la Educación Superior -ECAES en economía del 2007 y con otras fuentes secundarias. Específicamente, las encuestas se aplicaron a estudiantes de economía de 38 Instituciones de Educación Superior. Adicionalmente, se recopiló la información obtenida de las bases de datos suministradas por el ICFES. Contando las dos formas de recolección de información, se dispone en total con información de 3.404 estudiantes de Economía, quienes se encontraban en mayor proporción ( $73,75 \%$ de la muestra nacional) cursando últimos semestres durante el 2007.

En cuanto a las preguntas de la encuesta a los estudiantes, se realizaron de acuerdo a un ejercicio previo realizado en una universidad por Silva \& Sarmiento (2006), así como otras preguntas que resultaron relevantes adicionar desde la revisión de la literatura. La encuesta incluye preguntas sociodemográficas, acerca de la calidad de la educación secundaria, desenvolvimiento en la carrera, resultados académicos durante la trayectoria estudiantil, la participación en el mercado laboral y factores motivacionales.

A partir de la encuesta y de la información institucional se construyó la base de datos, para así realizar estadísticos descriptivos que permitieron explorar las principales características asociadas a la formación del economista en Colombia. En el Anexo 1 se presenta el universo de estudio de programas y estudiantes para cada una de las características analizadas en este documento, además se exponen las fuentes de información.

\section{Caracterización de los Departamentos, Facultades y Programas de Economía en Colombia}

La escasez de estudios que caractericen la formación de los estudiantes de economía en Colombia, tanto de las instituciones de educación superior como de los estudiantes, dificulta el diagnóstico de las problemáticas en la enseñanza de economía en Colombia. A continuación se describirán algunas características de los estudiantes, los programas de economía y las 60 universidades que los ofrecieron.

Esta sección comienza presentando las características de las instituciones de educación superior y los programas de economía, analizando la disponibilidad de recursos académicos y físicos, entre otros. Posteriormente, se realizará una caracterización del perfil de los estudiantes, para de esta forma comple-

\footnotetext{
6 La Resolución 2774 de 2003 establece que el componente económico o área de formación básica contempla las temáticas relacionadas a la investigación, los principios, las teorías y las políticas económicas. El componente profesional es compuesto por los conocimientos que "hacen viable la comprensión, interpretación, proyección y aplicación de las teorías y políticas económicas y su integración con las demás áreas del saber". El componente de énfasis se refiere al área de interés particular en que cada programa enfoca su plan de estudios. Por último, el componente socio-humanístico abordar las áreas que complementar la formación del economista y facilitan su conexión con las demás disciplinas del conocimiento.

7 En esta área se consideraron las asignaturas relacionadas con: Introducción a la economía, Microeconomía, Macroeconomía, Econometría, Historia Económica, Pensamiento Económico, Investigación.

8 En esta área se consideraron las asignaturas: Cuantitativas, Estadísticas, Administrativas, Contabilidad y Finanzas.

9 En esta área se consideraron las asignaturas: Socio-Humanísticas, derecho e idiomas.
} 
tar con la caracterización de la formación de economistas en Colombia.

\subsection{Caracterización Institucional}

El primer tópico de análisis sobre las instituciones que ofrecieron el programa de economía durante el 2007 en Colombia es la ubicación geográfica de éstas. En este sentido, se observa que los programas de economía se ofrecieron principalmente en la región occidental del país y que la mayor proporción de instituciones de educación superior se encontraban en Bogotá (44,83\%), seguidos de Cali $(10,22 \%)$ y Medellín (8,78\%), ciudades que son a su vez las que mayor población del país agrupan. En cifras, la mayoría de los departamentos no poseen o poseen como máximo 4 instituciones con esta oferta académica.

De las 60 instituciones analizadas en el 2007, el $23,33 \%$ eran de carácter oficial y el $76,67 \%$ privadas, de igual manera $21,67 \%$ contaban con la acreditación del programa de economía y el $16,67 \%$ con la acreditación institucional.

Al indagar por el valor de la matrícula de los programas de economía en el 2007 se encontró que el valor promedio de la matrícula de un estudiante de economía fue de aproximadamente 5 salarios mínimos de 2007, siendo 5,6 para el caso de las matrículas en las instituciones privadas y 1,3 en las públicas. La importancia de este componente de los programas de economía del país radica en el hecho de constituirse como una de las principales barreras de entrada a los mismos. La Tabla 3 expone algunos estadísticos descriptivos de los costos de la matrícula para el programa de economía de acuerdo a la institución.

Por último, los estudiantes de economía en el 2007 representaban aproximadamente el $4 \%$ del total de estudiantes inscritos en las instituciones de educación superior durante el 2007.
Tabla 3. Valor de matrícula programas de economía, salarios mínimos de 2007. Muestra nacional -200710

\begin{tabular}{|l|c|c|c|}
\hline \multicolumn{1}{|c|}{ Valor Matrícula } & Mínimo & Máximo & Promedio \\
\hline Total Instituciones & 0,61 & 18,83 & 4,95 \\
Instituciones Públicas & 0,61 & 4,40 & 1,31 \\
Instituciones Privadas & 1,70 & 18,83 & 5,61 \\
Instituciones Acreditadas & 3,07 & 18,83 & 8,43 \\
Instituciones No Acreditadas & 0,61 & 9,75 & 4,02 \\
\hline
\end{tabular}

\subsubsection{Recursos académicos}

Diferentes autores coinciden en señalar que a nivel mundial se ha venido presentando un crecimiento importante del sistema de educación superior, no sólo en el sentido un mayor número de estudiantes sino también debido a la mayor diversidad de programas y de instituciones de educación superior (Virgüez, Silva \& Sarmiento, 2013).

Para Gucht \& Westerheijden (1993) la importancia del estudio de la educación superior radica en la necesidad de probar las habilidades de las instituciones para la satisfacción de las necesidades y aspiraciones de los estudiantes en un entorno de constante cambio en el mercado laboral y de la sociedad en general. Por tanto, los factores institucionales han sido un elemento central de análisis en la literatura acerca de los componentes que influyen en la calidad de la educación superior (Woessmann, 2003; Montenegro, 2005; Valens, 2007).

Dentro de los factores institucionales considerados relevantes para la caracterización de la formación de los economistas, se indagó acerca de los recursos académicos con los que contaron los estudiantes. De los aspectos curriculares, tal y como se menciona en la sección 3 de este documento, se analizaron los planes de estudios por medio de las áreas de

10 Fuente. Sistema Nacional de Información de Educación Superior -SNIES, Ministerio de Educación Nacional (2008). Cálculos de los autores. 
formación básicas definidas en la Resolución 2774 de 2003 del Ministerio de Educación Nacional. De esta manera se encontró que en promedio el componente básico tiene 73 créditos, el profesional 44 y el socio-humanístico 22 . Adicionalmente, no se encontró diferencias significativas en el número de créditos entre programas acreditados y no acreditados.

En lo referente al componente de énfasis, la mayoría de las instituciones no hacen explícito qué característica específica quieren imprimirle a su programa de economía, limitándose a mencionar las características de los economistas graduados de cada programa para desempeñarse en el mercado laboral. No obstante, al analizar la misión, visión, perfil y plan de estudio de los programas, entre otros; se observa que varios de ellos están marcados por el énfasis en las Finanzas, Empresariado y por el sector Exterior/ Internacional (22 programas).

La Tabla 4 y 5 muestran algunos estadísticos para 53 planes de estudios, realizando una agrupación para 10 clases de asignaturas; encontrándose que en promedio se ofrecen carreras de 41-61 asignaturas y de 144-190 créditos. Se encontró que hay cierta homogeneidad en las instituciones en cuanto al número de asignaturas de Introducción a la Economía (1, 73,58\%), Microeconomía (2-4, 90,57\%), Estadística (2, 77,36\%), Econometría (2, 67,92\%) y Cuantitativas (4-5, 67,92\%). Del mismo modo, en cuanto a otras asignaturas complementarias a la formación integral del economista, en el 2007 los planes de estudio no contenían asignaturas como Idiomas $(56,60 \%)$ y Sistemas $(66,04 \%)$.

Sin embargo, no hay un consenso claro en el número de asignaturas como Macroeconomía (7-16), Historia Económica (0-3), Pensamiento Económico (0-2), Electivas de profundización (2-9), Contabilidad y Finanzas (2-8), Socio-humanísticas (3-10), Administrativas (0-3), Derecho (0-2), Investigación (0-4), Prácticas y/o Trabajos de Grado de carácter obligatorio (0-2). Por otro lado, se observa el surgimiento en algu- nos planes de estudio de por lo menos una asignatura referente al ámbito Ambiental y Ecología (37,74\%). Analizando el número de créditos, con el propósito de identificar la intensidad de los tópicos vistos, se observa también una alta variabilidad en cuanto a su cantidad; no obstante, el conjunto de mayor intensidad es en las asignaturas de Contabilidad y Finanzas, Cuantitativas, Electivas de profundización, Microeconomía, Macroeconomía, Socio-humanísticas y Prácticas y/o Trabajos de Grado. Además, el mayor número de asignaturas y créditos es para Macroeconomía.

Al implementar el sistema de créditos, algunas instituciones ya no operan con el concepto de semestre, por lo que no es posible determinar la duración de la carrera. Durante el 2007, de un total de 49 instituciones el mayor porcentaje de programas con carga completa posee una duración de la carrera de 10 semestres $(57,14 \%)$ seguido de 9 semestres $(34,69 \%)$ y $8(8,16 \%)$. En el caso de los programas acreditados, el mayor porcentaje cuenta con 9 semestres $(45,45 \%)$ seguido de $10(36,36 \%)$ y $8(18,18 \%)$; y en los no acreditados el mayor porcentaje cuenta con 10 semestres $(63,16 \%)$ seguido de $9(31,58 \%)$ y $8(5,26 \%)$.

Esta cifra es equivalente a la encontrada para Chile en el 2006, similar a la de Argentina que oscilaba entre cuatro y cinco años y medio, y a la de México donde la duración promedio de la carrera era de cuatro años y medio, con tendencia a la reducción (Espinoza, Machicado \& Markhlouf, 2009; Ahumada \& Butler, 2009; Rozenwurcel, Bezchinsky \& Rodríguez, 2009).

Dentro de los recursos académicos, también se encontraron diferencias en el número de monitores $^{11}$ desagregados por las áreas básicas de formación, teniendo en cuenta si las instituciones de la muestra nacional se encontraban acreditadas de alta calidad ${ }^{12}$ o no. En este sentido, se identificó que las instituciones con acreditación tuvieron un promedio mayor de

\footnotetext{
11 Este término se refiere a profesores complementarios o asistentes que en general resuelven dudas, talleres o ejercicios extra-clase.

12 La acreditación de alta calidad de universidades y programas de pregrado la otorga el Ministerio de Educación Nacional como una de las estrategias estatales para incentivar la calidad de la educación superior en Colombia.
} 
Tabla 4. Estadísticos descriptivos del número de asignaturas planes de estudios de economía - $2007^{13}$

\begin{tabular}{|c|c|c|c|c|c|c|c|}
\hline Asignatura* & Promedio & $\begin{array}{c}\text { Desviación Estándar } \\
\text { (D.E.) }\end{array}$ & Prom. - D.E. & $\begin{array}{l}\text { Prom. + } \\
\text { D.E. }\end{array}$ & Mínimo & Máximo & Moda \\
\hline Macroeconomía & 11,43 & 4,15 & 7,28 & 15,59 & 3 & 27 & 13 \\
\hline Microeconomía & 3,08 & 0,98 & 2,10 & 4,05 & 1 & 6 & 3 \\
\hline Estadística & 2,00 & 0,48 & 1,52 & 2,48 & 1 & 3 & 2 \\
\hline Econometría & 1,87 & 0,68 & 1,19 & 2,55 & 1 & 5 & 2 \\
\hline Historia Económica & 1,74 & 1,53 & 0,20 & 3,27 & 0 & 8 & 1 \\
\hline Pensamiento Económico & 1,04 & 0,92 & 0,12 & 1,96 & 0 & 4 & 1 \\
\hline Electivas & 5,10 & 3,63 & 1,46 & 8,73 & 0 & 15 & 3 \\
\hline Cuantitativas & 4,06 & 1,15 & 2,91 & 5,21 & 1 & 6 & 4,5 \\
\hline Contabilidad y Finanzas & 5,11 & 2,85 & 2,27 & 7,96 & 1 & 13 & 3,6 \\
\hline Socio-humanísticas & 6,64 & 3,29 & 3,35 & 9,94 & 0 & 17 & 6 \\
\hline Administrativas & 1,57 & 1,72 & 0,00 & 3,28 & 0 & 7 & 0 \\
\hline Ambiental y Ecología & 0,49 & 0,70 & 0,00 & 1,19 & 0 & 2 & 0 \\
\hline Derecho & 1,36 & 1,11 & 0,25 & 2,47 & 0 & 4 & 1 \\
\hline Idiomas & 1,68 & 2,32 & 0,00 & 4,00 & 0 & 8 & 0 \\
\hline Introducción a Economía & 1,09 & 0,56 & 0,53 & 1,66 & 0 & 3 & 1 \\
\hline Investigación & 1,79 & 2,10 & 0,00 & 3,89 & 0 & 8 & 0 \\
\hline Prácticas y/o trabajos de grado & 1,13 & 1,14 & 0,00 & 2,28 & 0 & 4 & 0 \\
\hline Sistemas & 0,62 & 1,06 & 0,00 & 1,68 & 0 & 4 & 0 \\
\hline Total asignaturas & 51,70 & 9,57 & 42,13 & 61,27 & 33 & 87 & 54 \\
\hline
\end{tabular}

monitores por área que aquellas instituciones que no se encontraban acreditadas (Ilustración 2). Este factor podría ser relevante porque es una de las variables que refleja los recursos académicos con que cuentan los estudiantes para un mayor acompañamiento y profundización en su formación como economistas.

Otros factores que se consideraron relevantes para el estudio de la evolución de los programas de economía, y que además puede ser visto como un factor de calidad para las instituciones de educación superior son los programas de posgrado, ya que permiten a los estudiantes opciones co-terminales quienes desde antes de graduarse pueden profundizar en determinados tópicos y vincularse con espacios de investigación.
Se observó que el 79,59\% de los programas poseen al menos un programa de posgrado, el $77,55 \%$ al menos una especialización, el $25 \%$ al menos una maestría y solo una institución un programa de doctorado en el 2007. Comparando si el programa está acreditado o no, se observa que ambos ofrecen en su gran mayoría los programas de especialización $(90,91 \%$ para acreditados y $73,68 \%$ para no acreditados), pero los programas acreditados ofrecen en mayor proporción algún programa de maestría (60\%) a diferencia de los no acreditados (15,79\%).

De acuerdo a la información obtenida, se evidenció que el 77,08\% de las instituciones contaban con al menos un grupo de investigación suscrito a las áreas

13 Fuente: Cálculos de los Autores. 
Tabla 5. Estadísticos descriptivos del número de créditos de las asignaturas de los planes de estudios de Economía $-2007^{14}$

\begin{tabular}{|c|c|c|c|c|c|c|c|c|}
\hline Asignatura & No. obs. & Promedio & $\begin{array}{l}\text { Desviación } \\
\text { Estándar }\end{array}$ & Prom. - D.E. & Prom. + D.E. & Mínimo & Máximo & Moda \\
\hline Macroeconomía & 35 & 38,89 & 14,02 & 24,86 & 52,91 & 12 & 77 & 28 \\
\hline Microeconomía & 35 & 11,40 & 3,07 & 8,33 & 14,47 & 4 & 18 & 12 \\
\hline Estadística & 35 & 6,71 & 1,84 & 4,87 & 8,55 & 3 & 11 & 6 \\
\hline Econometría & 35 & 6,86 & 2,17 & 4,69 & 9,03 & 4 & 12 & 8 \\
\hline Historia Económica & 27 & 7,15 & 5,04 & 2,11 & 12,18 & 2 & 20 & 4 \\
\hline Pensamiento Económico & 26 & 4,62 & 2,35 & 2,26 & 6,97 & 1 & 12 & 4 \\
\hline Electivas & 32 & 20,63 & 14,61 & 6,01 & 35,24 & 2 & 57 & 12 \\
\hline Cuantitativas & 35 & 14,69 & 3,82 & 10,87 & 18,50 & 6 & 22 & 15 \\
\hline Contabilidad y Finanzas & 35 & 15,83 & 8,77 & 7,06 & 24,60 & 3 & 41 & 12 \\
\hline Socio-humanísticas & 34 & 16,74 & 8,72 & 8,02 & 25,45 & 2 & 39 & 18 \\
\hline Administrativas & 19 & 7,47 & 4,13 & 3,35 & 11,60 & 2 & 15 & 8 \\
\hline Ambiental y Ecología & 15 & 3,60 & 1,18 & 2,42 & 4,78 & 2 & 6 & 4 \\
\hline Derecho & 29 & 4,14 & 2,60 & 1,54 & 6,74 & 1 & 11 & 2 \\
\hline Idiomas & 12 & 7,92 & 5,57 & 2,35 & 13,48 & 0 & 18 & $4,8,12$ \\
\hline Introducción a Economía & 31 & 4,48 & 1,96 & 2,52 & 6,45 & 1 & 12 & 4 \\
\hline Investigación & 24 & 7,42 & 6,63 & 0,79 & 14,04 & 1 & 31 & 4 \\
\hline Prácticas y/o trabajos de grado & 21 & 11,24 & 5,21 & 6,02 & 16,45 & 3 & 20 & 10 \\
\hline Sistemas & 12 & 3,83 & 2,29 & 1,54 & 6,12 & 0 & 8 & 4 \\
\hline Total créditos & 35 & 167,26 & 22,92 & 144,34 & 190,18 & 136 & 233 & 160,167 \\
\hline
\end{tabular}

económicas de los cuales el $43,75 \%$ se encontraban reconocidos en el 2007 por Colciencias.

De la misma forma, se indagó acerca del número de revistas que estaban adscritas a áreas económicas en la muestra nacional de instituciones, en la cual se identificó que el $51,11 \%$ de las instituciones no tenían revistas. Además, de las que tenían revistas tan sólo el $24,44 \%$ se encontraban indexadas por Colciencias.

\subsubsection{Profesores}

Aspectos relacionados a la cantidad, calidad y formación de los profesores, son materia importante de estudio cuando se trata de caracterizar la educación. La literatura presenta evidencia sobre los efectos positivos en el aprendizaje de los estudiantes del número de profesores (Kolpin \& Singel, 1996; Fligio, 1999; Borjas \& Acosta, 2000), así como su nivel educativo (Jepsen, 2005; Croninger, Rice, Rathbun \& Nishio, 2007; Fligio \& Kenny, 2007) y experiencia (Walstad \& Rebeck, 2001; Jepsen, 2005; Borman \& Kimball, 2005).

La información recolectada en el 2007 sobre el profesorado de los programas de economía en Colombia indica que en términos absolutos, en promedio había 6,87 docentes en el área de Macroeconomía, (mínimo tres docentes si el programa estaba acredi-

14 Fuente: Cálculos de los autores. 
Ilustración 2. Promedio de monitores por área según la situación de acreditación del programa de economía $-2007^{15}$

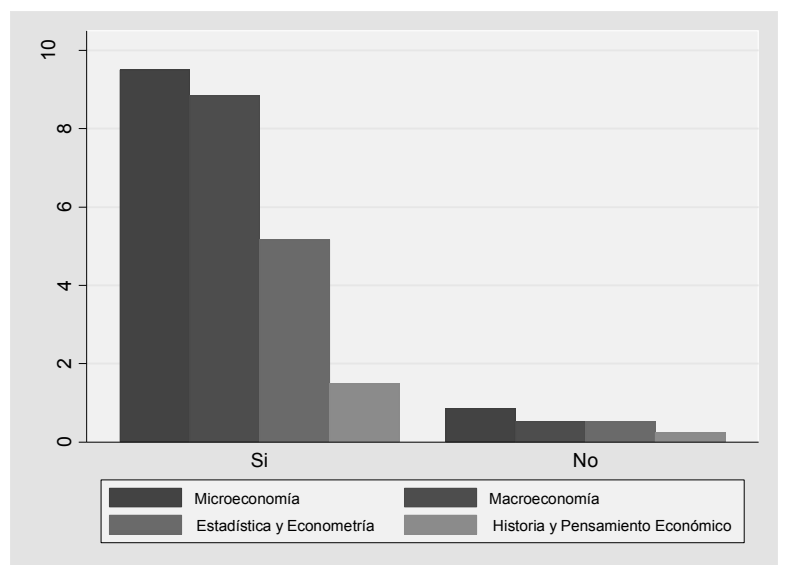

tado y uno si no lo estaba); 5,2 docentes relacionados con Microeconomía; 3,2 docentes en Estadística-Econometría (mínimo dos para los acreditados y uno para los no acreditados); y 3,6 docentes para Historia y Pensamiento Económico.

Discriminando por origen de la institución, es mayor el número promedio de docentes, tanto de planta como de cátedra, para los programas de economía de instituciones públicas por componente. En lo referente al sexo del profesor, hay un mayor porcentaje de docentes hombres que mujeres para cada área; respectivamente en el caso de Macroeconomía la relación es 79,61\% - 20,39\%, en Microeconomía es $79,49 \%$ - 20,51\%, Estadística y Econometría es $81,25 \%$ - 18,75\% e Historia y Pensamiento Económico es 75,93\% - 24,07\%. A excepción de Estadística - Econometría, esta relación de hombres es mayor en el caso de los programas de instituciones públicas y menor en las privadas.

En cuanto a la dedicación al programa, hay un mayor porcentaje de profesores de planta frente a los de cátedra en todas las áreas a excepción de Historia y Pensamiento Económico, respectivamente la relación es un $58 \%$ - $42 \%$ para las tres primeras.
Entre los profesores de planta, lo más común es desempeñarse como profesor de tiempo completo (por encima del $75 \%$ ), seguido de medio tiempo (hasta un $19 \%$ ) y lo menos frecuente es vincularse con dedicación exclusiva.

Respecto al nivel máximo de estudios alcanzado por los docentes hasta 2007, para Macroeconomía y Microeconomía generalmente se presentó una mayor proporción de docentes con estudios de maestría. En Estadística-Econometría predominaron los profesores con especialización, en cambio en Historia-Pensamiento la distribución es muy pareja entre estudios de pregrado, especialización y maestría, aunque permanece baja la proporción de profesores con doctorado. La Tabla 6 expone algunas de las principales características asociadas a los profesores de los programas de economía en Colombia.

Por otro lado, se presentó un número reducido de docentes con estudios de doctorado en los programas no acreditados a diferencia de los acreditados; no obstante lo que más se observó fue profesionales en los acreditados y maestros en los no acreditados. Se podría decir entonces que en los programas acreditados se contaba con un mayor volumen de profesores, pero con marcadas diferencias en su nivel de formación; mientras que en los no acreditados es menor el número de docentes pero con un similar nivel de estudios. Por otro lado, no se observan diferencias marcadas discriminando los programas por origen.

En cuanto a los docentes de tiempo completo, en los programas acreditados la mayor proporción tuvieron doctorado, mientras que en los no acreditados fue especialización; a nivel global se distribuye uniforme entre las cuatro categorías del nivel máximo de estudios. En contraste, entre los profesores de cátedra, los que tenían doctorado casi no prestaron sus servicios docentes; fue más común que los docentes de cátedra tuvieran solamente pregrado en los programas acreditados, mientras que en los no acreditados fueron más frecuentes los docentes de cátedra con especialización y maestría.

15 Fuente: Cálculos de los autores. 
Tabla 6. Principales características de los profesores por área de conocimiento - $2007^{16}$

\begin{tabular}{|l|c|c|c|c|}
\hline & Macroeconomía & Microeconomía & $\begin{array}{c}\text { Estadística y } \\
\text { econometría }\end{array}$ & $\begin{array}{c}\text { Historia y pensamiento } \\
\text { económico }\end{array}$ \\
\hline Hombres (\%) & 79,61 & 79,49 & 81,25 & 75,93 \\
Mujeres (\%) & 20,39 & 20,51 & 18,75 & 24,07 \\
Profesores de planta (\%) & 58,97 & 76,92 & 35,90 & 20,51 \\
Profesores de catedra (\%) & 41,03 & 55,13 & 25,64 & 48,72 \\
Profesores profesional (\%) & 28,21 & 28,21 & 14,10 & 20,51 \\
Profesores especialización (\%) & 14,10 & 38,46 & 20,51 & 21,79 \\
Profesores maestría (\%) & 44,87 & 57,69 & 17,95 & 20,51 \\
Profesores doctorado (\%) & 12,82 & 7,69 & 8,97 & 6,41 \\
Promedio por programa oficiales & 7,8 & 3,4 & 2,6 & 2,8 \\
Promedio por programa privadas & 6,4 & 6,1 & 3,5 & 4 \\
Promedio por programa total & 6,87 & 5,2 & 3,2 & 3,6 \\
\hline
\end{tabular}

\subsubsection{Recursos administrativos y físicos}

Los recursos administrativos y físicos dan cuenta de la infraestructura a disposición de los estudiantes para su proceso de aprendizaje. Específicamente, en cuanto a los recursos administrativos necesarios para la gestión de la formación integral del economista, durante 2007 en promedio 349 personas estaban dedicadas a las áreas administrativas en las instituciones y 6 dedicadas a las del programa. En cuanto al personal dedicado al bienestar, salud física y psicológica en promedio fueron 22 personas.

De otro lado, los recursos bibliográficos con los que cuentan los estudiantes para su consulta en las instituciones se constituyen en uno de los insumos básicos para su formación (Becker \& Watts, 2001). Es así, como se evidenció que las instituciones de educación superior en promedio contaron con 1.244 libros de economía, siendo la mayor proporción de libros del área de Microeconomía y la menor de Estadística y Econometría. Asimismo, el promedio de libros en las instituciones acreditadas (2.969) es con- siderablemente superior al promedio de las no acreditadas (691). Estos hallazgos por área de formación se exponen en la Ilustración 3.

En el análisis de los recursos físicos, la proporción del número promedio de computadores disponibles por estudiante en las instituciones fue mayor en las instituciones acreditadas $(0,10$ ó un computador para 10 estudiantes) que en las no acreditadas ( 0,05 ó un computador para 20 estudiantes).

\subsection{Caracterización estudiantil}

Como se mencionó previamente, para realizar la caracterización estudiantil se aplicó una encuesta a los alumnos que estaban cursando estudios de economía durante el año 2007, así como se recopiló información del formato de inscripción al Examen de Calidad de la Educación Superior -Ecaes en economía del 2007 y de otras fuentes secundarias; obteniéndose una base de datos de 3.404 estudiantes de 60 instituciones. La descripción del perfil de los estudiantes se encuentra a continuación.

16 Fuente: Cálculos de los autores. 
Ilustración 3. Promedio de libros por área según la situación de acreditación del programa de economía $-2007^{17}$

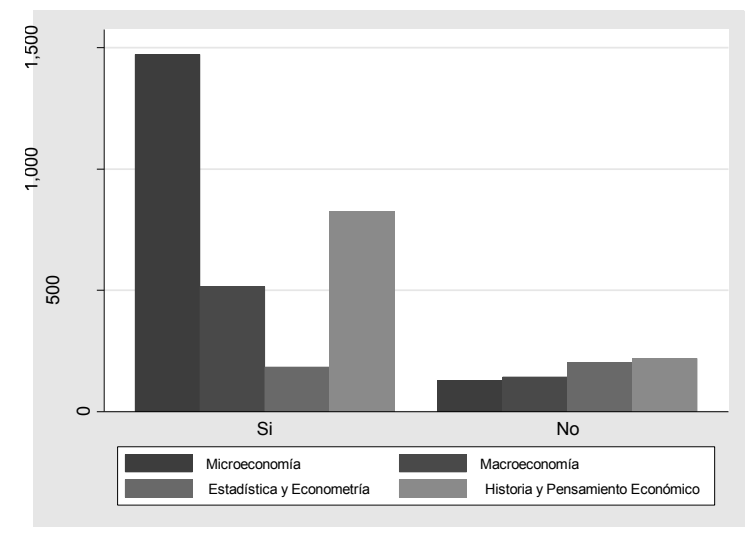

\subsubsection{Factores socioeconómicos}

Los factores asociados a los estudiantes suelen ser aspectos determinísticos de su formación, especialmente cuando se consideran factores socio-económicos. Dentro de las características analizadas se encontró que el 57,04\% de los estudiantes de economía eran mujeres y el $42,96 \%$ eran hombres. Esta composición diferiría de lo encontrado en otras investigaciones para estudiantes de América Latina, dado que las mujeres para el caso de los programas de posgrado representaron el 29\% para Estados Unidos en 1985 y el $41 \%$ para Chile en el período 20062007 (Colander \& Kramel, 1987; Colander \& Nopo, 2011). En el caso de los programas de pregrado, las mujeres representaron entre el $40 \%$ y el $50 \%$ de los estudiantes en Argentina para la década de 19952005 (Rozenwurcel, Bezchinsky \& Rodríguez, 2009), el 40\% en México para el 2006 (Ahumada \& Buttler, 2009) y el $31,2 \%$ en Chile para el 2007 (Espinoza, Machicado \& Makhlouf, 2009).

Como se mencionó anteriormente, la muestra se trata en su mayoría de estudiantes de últimos semestres de la carrera; encontrándose que se ubicaban principalmente en un rango de edades entre 16 y 30 años, con una concentración mayor de alumnos entre 16 y 25 años equivalente a un 76,05\% de la población analizada y alumnos entre 26 y 30 equivalente a un $17.08 \%$; siendo la edad promedio aproximadamente de 24 años (Ilustración 4).

En contraste, el $90 \%$ de los estudiantes de pregrado en Chile estuvieron entre los 20 y 24 años (Espinoza, Machicado \& Makhlouf, 2009); en México los estudiantes de pregrado de economía eran de rangos de edades más altos, ya que la proporción con menos de 30 años de edad era de $31 \%$ en el 2006, mientras que la de mayores de 50 años era de $24 \%$ en el mismo periodo (Ahumada \& Butler, 2009); en Argentina el promedio de edad de los estudiantes de las tres Universidades analizadas fue de 22 años (Rozenwurcel, Bezchinsky \& Rodríguez, 2009).

Otro aspecto a señalar, es que tal y como se observa en la Ilustración 5 , en la muestra nacional predominaron los estratos 2, 3 y 4, los cuales pueden ser enmarcados dentro de los estratos considerados para la población de clase media baja a media alta en Colombia. En este sentido, para el caso de los estudiantes de posgrado de Estados Unidos y Chile predominó la clase media y la clase media alta respectivamente (Colander \& Kramel, 1987; Colander $\&$ Nopo, 2011).

Adicionalmente, se estableció que para la muestra total el nivel máximo de escolaridad predominante

Ilustración 4. Estudiantes programas de economía. Muestra nacional por género y edad $-2007^{18}$

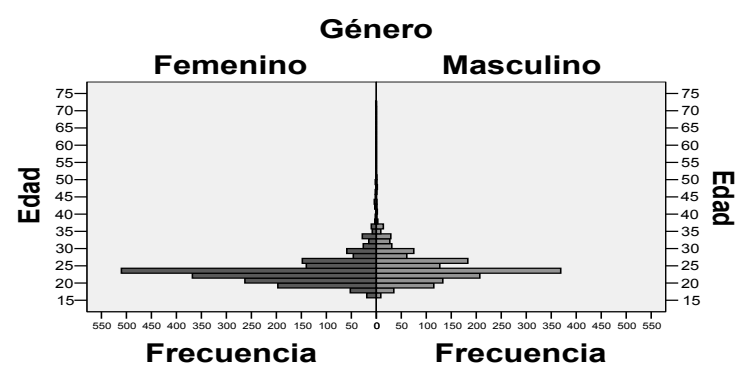

Fuente: Cálculos de los autores.

18 Fuente: Cálculos de los autores. 
Ilustración 5. Estrato socioeconómico estudiantes de economía. Comparativo nacional y carácter de la Institución -2007.19

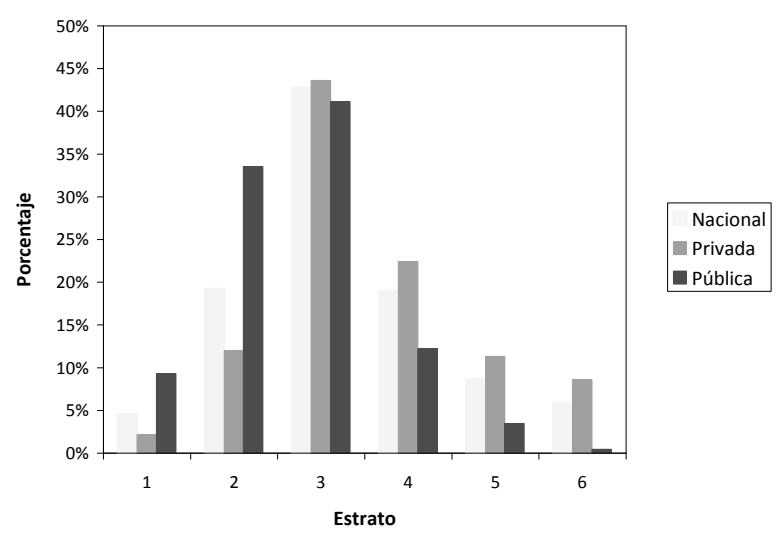

de los padres fue posgrado $(29,93 \%$ padre, $25,05 \%$ madre), y secundaria $(27,84 \%$ padre, $34,91 \%$ madre) como se muestra en la Tabla 7. Para efectos comparativos, se contaron como profesionales el $23 \%$ de las madres y el $28 \%$ de los padres de los estudiantes de economía en Bolivia. Asimismo, el $25 \%$ de los padres de estudiantes de pregrado mexicanos se contaron como profesionales (Colander \& Kramel, 1987; Colander \& Nopo, 2007; Ahumada \& Butler, 2009; Espinoza, Machicado \& Markhlouf, 2009).

Por otro lado, el estado conyugal del estudiante, ser cabeza de familia y el número de personas que tiene a cargo son factores que en cierta forma podrían indicar las responsabilidades adquiridas por el estudiante por fuera de la institución. Los resultados de la muestra nacional indicaron que el 91,25\% eran estudiantes solteros, seguidos por un $6,54 \%$ que se encontraban casados o en unión libre; el 7,35\% eran cabeza de familia y el $11,24 \%$ tenían por lo menos un hijo (Tabla 8). Adicionalmente, la mayoría de hogares estaban compuestos por entre tres y seis miembros $(83,40 \%)$, siendo la mayor proporción los hogares compuestos por cuatro personas $(30,70 \%)$.
Tabla 7. Nivel máximo de educación de los padres, distribución porcentual. Muestra nacional $-2007 .{ }^{20}$

\begin{tabular}{|c|c|c|}
\hline Nivel & Padre & Madre \\
\hline Ninguno & 1,06 & 0,63 \\
Preescolar & 0,49 & 0,48 \\
Básica Primaria & 19,4 & 18,07 \\
Secundaria & 27,84 & 34,91 \\
Media Técnica & 7,01 & 8,72 \\
Normalista & 0,27 & 1,16 \\
Tecnológica & 5,16 & 4,54 \\
Profesional & 8,83 & 6,45 \\
Posgrado & 29,93 & 25,05 \\
\hline
\end{tabular}

Tabla 8. Responsabilidades familiares -Muestra nacional $2007^{21}$

\begin{tabular}{|c|c|c|}
\hline \multicolumn{2}{|c|}{ Característica } & $\begin{array}{l}\text { Proporción de } \\
\text { estudiantes (\%) }\end{array}$ \\
\hline \multicolumn{2}{|l|}{ Solteros } & 91,25 \\
\hline \multicolumn{2}{|c|}{ Casados o unión libre } & 6,54 \\
\hline \multicolumn{2}{|c|}{ Viudos, separados o divorciados } & 2,21 \\
\hline \multicolumn{2}{|c|}{ Tienen al menos un hijo } & 11,24 \\
\hline \multicolumn{2}{|c|}{ Son Cabeza de familia } & 7,35 \\
\hline \multirow{7}{*}{$\begin{array}{c}\text { Cantidad de miembros en } \\
\text { el hogar }\end{array}$} & 1 & 1,74 \\
\hline & 2 & 7,96 \\
\hline & 3 & 17,96 \\
\hline & 4 & 30,7 \\
\hline & 5 & 24,3 \\
\hline & 6 & 10,44 \\
\hline & 70 más & 6,9 \\
\hline
\end{tabular}

Otra variable a analizar es el ingreso familiar de los estudiantes, factor para el cual se establecieron nueve intervalos de respuesta según el salario mínimo (SM) mensual legal vigente en el 2007. De los resultados obtenidos a nivel nacional y presentados en la Tabla 9, se pudo establecer que existía una concentración en el intervalo entre 2 y $3 \mathrm{SM}$, equivalente al 26,19\% de la muestra. Es de resaltar que estos

\footnotetext{
9 Fuente: Cálculos de los autores.

20 Fuente: Cálculos de los autores.

21 Fuente: Cálculos de los autores.
} 
ingresos correspondieron en su mayoría al aporte de dos personas en el hogar (46,45\%).

Tabla 9. Nivel de ingresos familiares de los estudiantes de economía. Muestra nacional -2007.22

\begin{tabular}{|l|c|c|c|}
\hline \multicolumn{1}{|c|}{ Ingreso del Hogar } & Frecuencia & Porcentaje & $\begin{array}{c}\text { Porcentaje } \\
\text { acumulado }\end{array}$ \\
\hline Menos de un SM & 100 & 6,42 & 6,42 \\
Entre 1 y menos de 2 SM & 249 & 15,98 & 22,4 \\
Entre 2 y menos de 3 SM & 408 & 26,19 & 48,59 \\
Entre 3 y menos de 5 SM & 259 & 16,62 & 65,21 \\
Entre 5 y menos de 7 SM & 241 & 15,47 & 80,68 \\
Entre 7 y menos de 9 SM & 63 & 4,04 & 84,72 \\
Entre 9 y menos de 11 SM & 77 & 4,94 & 89,67 \\
Entre 11 y menos de 13 SM & 55 & 3,53 & 93,2 \\
13 SM en adelante & 106 & 6,8 & 100 \\
Total & 1,558 & 100 & \\
\hline
\end{tabular}

Finalmente, teniendo en cuenta los estudiantes de economía pertenecientes a algún grupo étnico, la mayor representación se presenta entre los afrocolombianos $(70 \%)$ seguidos por los indígenas $(26 \%)$.

\subsubsection{Educación secundaria}

La educación secundaria es un factor importante en la determinación de éxito de los estudiantes en la educación superior (Eskew \& Faley, 1998). Es por esto que se consideró relevante brindar una caracterización acerca de los colegios en los cuales se llevó a cabo este proceso de formación, permitiendo a su vez tener un perfil más amplio del historial académico de los estudiantes previo a la realización de sus estudios de educación superior. Además, se analizaron los resultados del Examen del Estado para acceso a la Educación Superior - ICFES con el fin evidenciar preliminarmente el nivel académico y las dotaciones iniciales de conocimiento de los estudiantes de economía.

Así, se encontró que un $56,51 \%$ de los estudiantes de la muestra nacional estuvieron vinculados a co- legios de carácter privado y un $41,02 \%$ a públicos; también se encontró que el $85,06 \%$ pertenecían a colegios de Calendario A y que sólo el 0,49\% estudió en la noche (Tabla 10). Además, el título de educación secundaria obtenido por los estudiantes se distribuyó entre académico (81,72\%), Técnico $(16,33 \%)$ y Normalista $(1,95 \%)$.

Tabla 10. Características de la educación secundaria Muestra nacional $2007^{23}$

\begin{tabular}{|c|c|c|}
\hline \multicolumn{2}{|c|}{ Características del Colegio } & $\begin{array}{c}\text { Proporción de } \\
\text { estudiantes (\%) }\end{array}$ \\
\hline \multirow{3}{*}{ Tipo } & Público & 41,02 \\
& Privado & 56,51 \\
& Conseción & 2,47 \\
\hline \multirow{3}{*}{ Calendario } & Calendaría A & 85,06 \\
& Calendario B & 14,57 \\
& Otro & 0,37 \\
\hline \multirow{3}{*}{ Jornada } & Completa & 18,08 \\
& Diurna & 67,06 \\
& Tarde & 14,37 \\
& Nocturno & 0,49 \\
\hline \multirow{2}{*}{ Título } & Académico & 81,72 \\
& Normalista & 1,95 \\
& Técnico & 16,33 \\
\hline
\end{tabular}

De igual forma se pudo evidenciar que el puntaje total promedio del ICFES de la muestra nacional de estudiantes de economía fue de 52,25 puntos, siendo para la prueba de matemáticas 48,83 y para la de lenguaje 56,01 (Ilustración 6).

\subsubsection{Desenvolvimiento en la carrera}

Otras variables que se podrían analizar de manera paralela a los estudios que realizan los estudiantes, son aquellas que se refieren a la dedicación que han tenido en el desarrollo de su carrera y a la financiación a la que han recurrido para llevar a cabo sus estudios.

22 Fuente: Cálculos de los autores.

23 Fuente: Cálculos de los autores. 
Ilustración 6. Resultados Icfes estudiantes de economía. Muestra nacional -2007.24

Total promedio simple

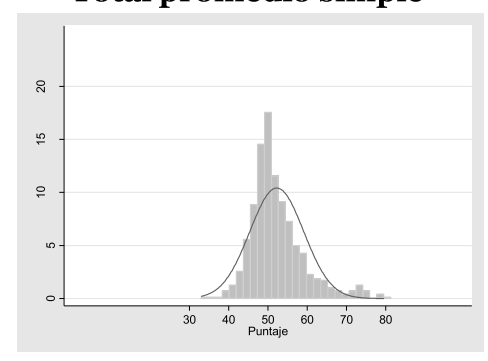

Matemáticas

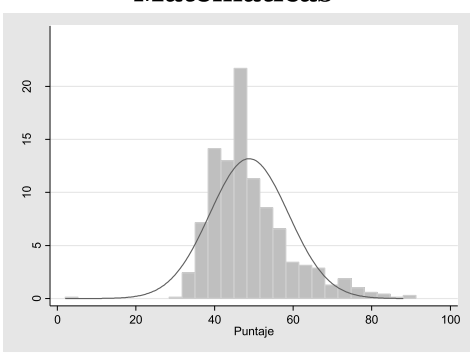

Lenguaje

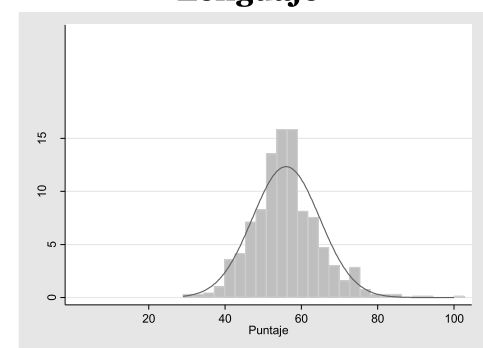

De esta manera, se evidenció en la muestra nacional que el 58,33\% de los recursos los proporcionó la familia del estudiante, seguido de un $17,10 \%$ que recurrieron a créditos (Tabla 11). En contraste con la evidencia internacional, en México cerca del $25 \%$ de los universitarios que estudiaron economía recibieron apoyo económico en forma de becas, cuyos requisitos para su obtención y mantenimiento varían siendo los más importantes el tener la necesidad económica y el mantener un alto desempeño académico (Ahumada \& Butler, 2009). En Bolivia el $88 \%$ de los estudiantes se financiaron con recursos familiares, tan sólo el $2,8 \%$ mencionó que recibió ayuda del gobierno y el $8,3 \%$ mencionó que su educación fue gratuita; en Chile, la gran mayoría de estudiantes de pregrado $(73 \%)$ se financiaron con recursos propios, el 1,8\% tenía beca y el $8,5 \%$

Tabla 11. Forma de financiación principal durante la mayor parte de sus estudios en economía. Muestra nacional $-2007.25$

\begin{tabular}{|c|c|}
\hline Forma & Porcentaje \\
\hline Beca académica & 4,85 \\
Crédito & 17,1 \\
Empresa donde labora & 2,24 \\
Familiar & 58,33 \\
Propia & 14,05 \\
Otra & 3,42 \\
\hline
\end{tabular}

tenía crédito universitario (Espinoza, Machicado \& Mackhlouf, 2009).

Para las actividades de estudio independiente, los alumnos de la muestra nacional tomaron aproximadamente 87 minutos a la semana, por cada hora de clase. Además en promedio acudieron a bibliotecas o hemerotecas 3 veces por semana.

Al analizar si los estudiantes tenían otro tipo de formación antes de terminar sus estudios de economía, se encontró que el 19,32\% ya poseía algún título técnico o profesional, que el 10,89\% estaba realizando otros estudios técnicos o profesionales paralelos a los de economía y que el 20,37\% ya habían empezado otra carrera y no lo habían terminado. Asimismo, el $5,96 \%$ había comenzado estudios en economía en otra institución diferente a la que se encontraba en el 2007.

\subsubsection{Resultados académicos durante la trayectoria estudiantil}

El desempeño de los estudiantes durante su trayectoria estudiantil es evaluado a partir del Promedio General Acumulado (PGA) alcanzado durante el transcurso de su carrera. Adicionalmente se analizan los resultados en el Examen de Calidad de la Educación Superior Ecaes (ahora denominado Saber Pro) implementado por el Icfes, el cual evaluaba el grado de desarrollo de las competencias Interpretativa, Argumentativa y Propositiva, de los estudiantes de úl-

24 Fuente: Cálculos de los autores.

25 Fuente: Cálculos de los autores. 
timos semestres de economía y el cual permite analizar, de manera uniforme, el nivel de conocimientos adquiridos por los estudiantes de economía, al ser una prueba estandarizada. En el 2007 la prueba estaba compuesta por cuatro componentes económicos (Macroeconomía, Microeconomía, Estadística y Econometría, Historia y Pensamiento Económico), un componente de Comprensión Lectora y uno de Inglés. Los resultados eran normalizados para que siempre el promedio nacional sea de aproximadamente 10 puntos con una desviación estándar de 1 (para una discusión acerca de las características de esta prueba, ver Sarmiento \& Sandoval, 2008).

Los estudiantes de economía analizados tienen en promedio un PGA simple de 3,84 cuando se tiene en cuenta el total de las materias sin realizar ninguna ponderación (promedio simple-total), mientras que cuando se calcula el PGA ponderando de acuerdo a la cantidad de créditos que posee cada materia (promedio ponderado -total) el promedio de los estudiantes es de 3,83. Al analizar el PGA pero únicamente para la materias clasificadas en los componentes que fueron evaluados en el Ecaes (Macroeconomía, Microeconomía, Estadística y Econometría, Historia y Pensamiento Económico), tanto el promedio simple (promedio simple -componentes Ecaes) como el promedio ponderado (promedio ponderado -componentes Ecaes) se ubican en 3.76 puntos.

Tabla 12. Estadísticas PGA estudiantes de economía $-2007 .{ }^{26}$

\begin{tabular}{|l|c|c|c|c|c|}
\hline \multicolumn{1}{|c|}{ Variable } & $\begin{array}{c}\text { No. } \\
\text { obs. }\end{array}$ & Promedio & $\begin{array}{c}\text { Desv, } \\
\text { Est, }\end{array}$ & Min & Max \\
\hline Promedio simple-total & 191 & 3,84 & 0,30 & 2,96 & 4,62 \\
$\begin{array}{l}\text { Promedio simple- } \\
\text { componentes Ecaes }\end{array}$ & 216 & 3,76 & 0,36 & 2,82 & 4,74 \\
$\begin{array}{l}\text { Promedio ponderado- } \\
\text { total }\end{array}$ & 191 & 3,83 & 0,30 & 2,96 & 4,62 \\
$\begin{array}{l}\text { Promedio ponderado- } \\
\text { componentes Ecaes }\end{array}$ & 216 & 3,76 & 0,37 & 2,85 & 4,74 \\
\hline
\end{tabular}

26 Fuente: Instituciones de Educación Superior. Cálculos de los Autores.

27 Fuente: Icfes (2008). Cálculos de los Autores.
La tabla 12 expone estos resultados además de algunos otros estadísticos descriptivos sobre el PGA de los estudiantes de economía.

La evidencia para el caso Chileno, mostró que la mayor parte de los estudiantes tuvo un buen desenvolvimiento académico, logrando dos tercios de ellos un promedio acumulado en la carrera de 5 puntos ó más (en una escala de $1 \mathrm{al} 7$ ), con una fuerte concentración $(51,8 \%)$ en el tramo entre 5 y 5,4 puntos (Espinoza, Machicado \& Makhlouf, 2009).

De los resultados Ecaes, se observa que los estudiantes de instituciones de educación superior de carácter público y los de jornada diurna obtienen en promedio un puntaje total superior a la media nacional, contrario a lo que ocurre con los privados y nocturnos (Ilustración 7). Resultados similares a estos fueron hallados por Ortiz (2005) y Montenegro (2005) a partir del análisis de los resultados Ecaes de 2004, en contraste para este mismo año Meisel \& Pérez (2005) no encontraron mayores diferencias en el puntaje alcanzado por los estudiantes de las instituciones de educación superior de acuerdo a su origen privado o público.

Ilustración 7. Resultados Ecaes según el origen y jornada del programa en el que estudió $-2007 .{ }^{27}$

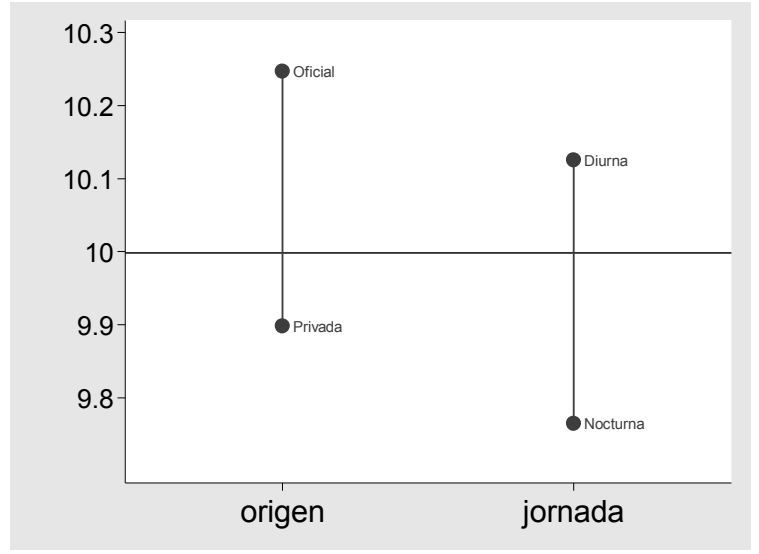


Igualmente, se observa un mejor desempeño en el Ecaes de los estudiantes de instituciones acreditadas tanto en términos absolutos como relativos; aunque el número de estudiantes de programas acreditados es menor, en todos los componentes poseen el mayor número de estudiantes en el nivel de desempeño alto, exceptuando el componente de comprensión lectora (Tabla 13).

Analizando en términos relativos, sin importar el tamaño de la muestra total, los estudiantes de programas acreditados presentan menos número de estudiantes con desempeño bajo que las no acreditadas (Ilustración 8)

Ilustración 8. Desempeño estudiantes en el Ecaes en economía -2007 según criterio de acreditación del programa. Términos relativos por componente y competencia evaluada ${ }^{28}$
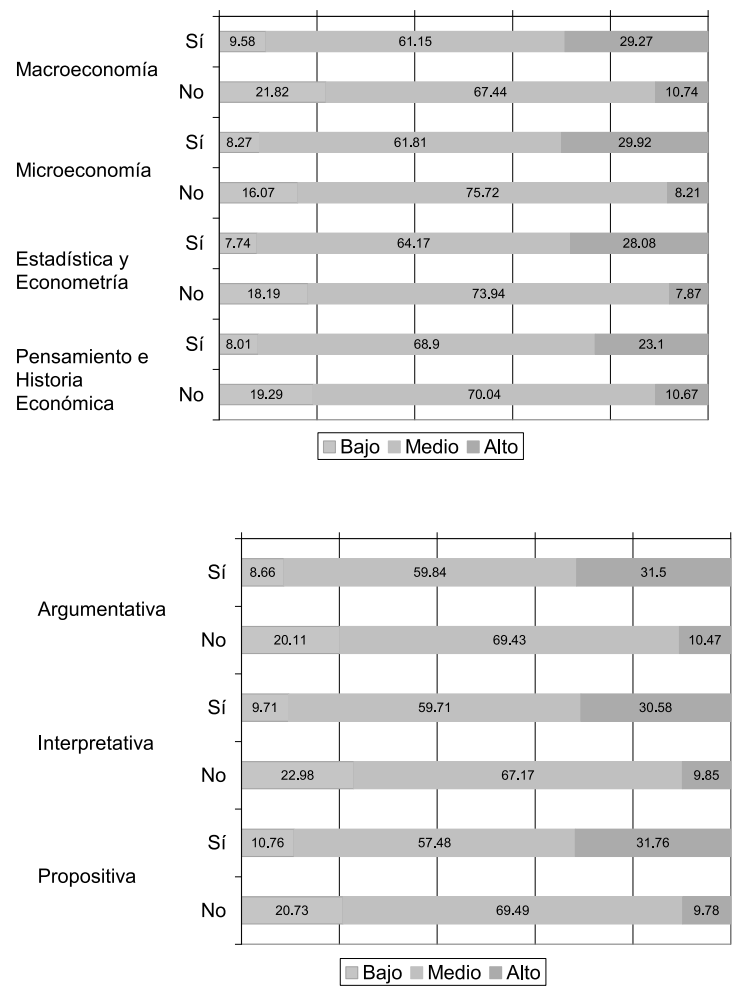

Tabla 13. Desempeño estudiantes en el Ecaes en economía -2007 según criterio de acreditación del programa ${ }^{29}$

\begin{tabular}{|c|c|c|c|c|}
\hline Componente & $\begin{array}{l}\text { Desempeño } \\
\text { en la prueba }\end{array}$ & $\begin{array}{l}\text { Programa } \\
\text { Acreditado }\end{array}$ & $\begin{array}{l}\text { Programa } \\
\text { No } \\
\text { acreditado }\end{array}$ & Total \\
\hline \multirow{3}{*}{$\begin{array}{c}\text { Macroecono- } \\
\text { mía }\end{array}$} & Bajo & 3,28 & 14,34 & 17,63 \\
\hline & Medio & 20,95 & 44,33 & 65,29 \\
\hline & Alto & 10,03 & 7,06 & 17,09 \\
\hline \multirow{3}{*}{$\begin{array}{l}\text { Microecono- } \\
\text { mía }\end{array}$} & Bajo & 2,83 & 10,57 & 13,4 \\
\hline & Medio & 21,18 & 49,78 & 70,95 \\
\hline & Alto & 10,25 & 5,4 & 15,65 \\
\hline \multirow{3}{*}{$\begin{array}{l}\text { Estadística y } \\
\text { Econometría }\end{array}$} & Bajo & 2,65 & 11,96 & 14,61 \\
\hline & Medio & 21,99 & 48,61 & 70,59 \\
\hline & Alto & 9,62 & 5,17 & 14,79 \\
\hline \multirow{3}{*}{$\begin{array}{l}\text { Pensamiento } \\
\text { e Historia } \\
\text { Económica }\end{array}$} & Bajo & 2,74 & 12,68 & 15,42 \\
\hline & Medio & 23,61 & 46,04 & 69,65 \\
\hline & Alto & 7,91 & 7,01 & 14,93 \\
\hline \multirow{5}{*}{ Inglés } & Nivel inferior A- & 0,99 & 6,65 & 7,64 \\
\hline & $\begin{array}{c}\text { Usuario básico } \\
\text { A1 }\end{array}$ & 5,04 & 23,74 & 28,78 \\
\hline & $\begin{array}{c}\text { Usuario básico } \\
\text { A2 }\end{array}$ & 5,53 & 14,66 & 20,19 \\
\hline & $\begin{array}{c}\text { Usuario } \\
\text { independiente } \\
\text { B1 }\end{array}$ & 11,29 & 16,46 & 27,74 \\
\hline & $\begin{array}{c}\text { Usuario } \\
\text { independiente } \\
\text { B+ }\end{array}$ & 11,42 & 4,23 & 15,65 \\
\hline \multirow{3}{*}{$\begin{array}{c}\text { Comprensión } \\
\text { Lectora }\end{array}$} & Bajo & 1,71 & 4,36 & 6,07 \\
\hline & Medio & 25,13 & 50,09 & 75,22 \\
\hline & Alto & 7,42 & 11,29 & 18,71 \\
\hline \multirow{3}{*}{$\begin{array}{l}\text { Competencia } \\
\text { Argumentativa }\end{array}$} & Bajo & 2,97 & 13,22 & 16,19 \\
\hline & Medio & 20,5 & 45,64 & 66,14 \\
\hline & Alto & 10,79 & 6,88 & 17,67 \\
\hline \multirow{3}{*}{$\begin{array}{l}\text { Competencia } \\
\text { Interpretativa }\end{array}$} & Bajo & 3,33 & 15,11 & 18,44 \\
\hline & Medio & 20,46 & 44,15 & 64,61 \\
\hline & Alto & 10,48 & 6,47 & 16,95 \\
\hline \multirow{3}{*}{$\begin{array}{l}\text { Competencia } \\
\text { Propositivo }\end{array}$} & Bajo & 3,69 & 13,62 & 17,31 \\
\hline & Medio & 19,69 & 45,68 & 65,38 \\
\hline & Alto & 10,88 & 6,43 & 17,31 \\
\hline
\end{tabular}

28 Fuente: Icfes (2008). Cálculos de los Autores.

29 Fuente: Icfes (2008) Cálculos de los Autores. Las cifras se encuentran en términos absolutos por componente y competencia evaluada. 
Al analizar la correlación existente entre el PGA y los resultados Ecaes, se encuentra una correlación positiva superior al 0,22 para cada componente del Ecaes emparejado con su equivalente del PGA, aunque no significativo para el caso de Microeconomía. La mayor correlación es entre el componente Pensamiento-Historia del Ecaes y Macroeconomía del PGA $(0,39)$. Evaluando la correlación entre todos los componentes por indicador de desempeño académico, existe una correlación superior a 0,49 para el Ecaes, siendo la mayor para Macroeconomía y Microeconomía $(0,69)$ y la menor para Estadística-Econometría y Pensamiento-Historia Económica $(0,49)$. Para el Pga, la mayor correlación es entre el componente Pensamiento-Historia y Macroeconomía $(0,69)$ y la menor es entre Pensamiento-Historia y Microeconomía $(0,35)$. Estos resultados se evidencian en la Tabla 14.

Tabla 14. Correlaciones Ecaes-PGA por componente -2007.30

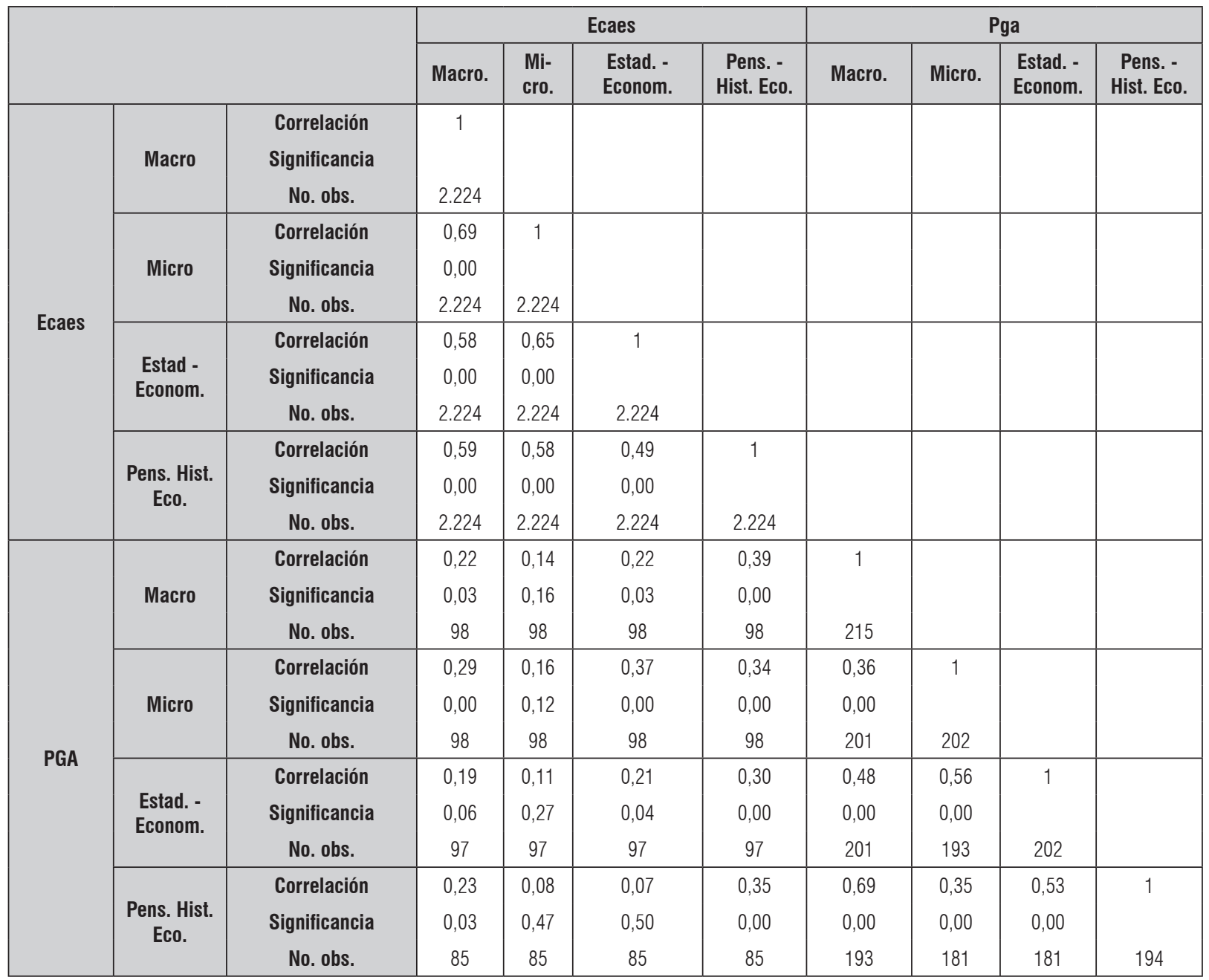

30 Fuente: Icfes (2008). Cálculos de los Autores. 
Cuando al análisis de los resultados académicos durante la trayectoria del estudiante de economía se incluyen el desempeño de éstos en la educación media (a partir de los resultados del Icfes), se observa una correlación positiva tanto del Pga total como del Icfes frente al Ecaes total, siendo superior al 0.33 para el
Pga, igual al 0,23 para el Icfes y al 0,16 para los componentes de matemáticas y lenguaje del Icfes; para todos los casos las correlaciones son significativas al $5 \%$. Por otro lado, no son significativas las correlaciones entre el Icfes y el PGA (Tabla 15).

Tabla 15. Correlaciones Icfes-PGA-Ecaes -2007. ${ }^{31}$

\begin{tabular}{|c|c|c|c|c|c|c|c|c|}
\hline & & & \multicolumn{2}{|c|}{ Ecaes } & \multicolumn{4}{|c|}{ PGA } \\
\hline & & & $\begin{array}{l}\text { Pro- } \\
\text { medio } \\
\text { simple }\end{array}$ & $\begin{array}{l}\text { Promedio } \\
\text { ponderado }\end{array}$ & $\begin{array}{l}\text { Promedio } \\
\text { simple - total }\end{array}$ & $\begin{array}{l}\text { Promedio } \\
\text { simple - } \\
\text { componen- } \\
\text { tes Ecaes }\end{array}$ & $\begin{array}{c}\text { Promedio } \\
\text { ponderado } \\
\text { - total }\end{array}$ & $\begin{array}{c}\text { Promedio } \\
\text { ponderado } \\
\text { componen- } \\
\text { tes Ecaes }\end{array}$ \\
\hline \multirow{12}{*}{ PGA } & \multirow{3}{*}{$\begin{array}{c}\text { Promedio } \\
\text { simple - total }\end{array}$} & Correlación & 0,41 & 0,39 & & & & \\
\hline & & Significancia & 0,00 & 0,00 & & & & \\
\hline & & No. obs. & 97 & 97 & & & & \\
\hline & \multirow{3}{*}{$\begin{array}{c}\text { Promedio } \\
\text { simple - } \\
\text { componentes }\end{array}$} & Correlación & 0,35 & 0,33 & & & & \\
\hline & & Significancia & 0,00 & 0,00 & & & & \\
\hline & & No. obs. & 99 & 99 & & & & \\
\hline & \multirow{3}{*}{$\begin{array}{c}\text { Promedio } \\
\text { ponderado - } \\
\text { total }\end{array}$} & Correlación & 0,40 & 0,38 & & & & \\
\hline & & Significancia & 0,00 & 0,00 & & & & \\
\hline & & No. obs. & 97 & 97 & & & & \\
\hline & \multirow{3}{*}{$\begin{array}{c}\text { Promedio } \\
\text { ponderado - } \\
\text { componentes }\end{array}$} & Correlación & 0,35 & 0,33 & & & & \\
\hline & & Significancia & 0,00 & 0,00 & & & & \\
\hline & & No. obs. & 99 & 99 & & & & \\
\hline \multirow{9}{*}{$\begin{array}{c}\text { Icfes } \\
\text { Base } \\
100\end{array}$} & \multirow{3}{*}{$\begin{array}{l}\text { Promedio } \\
\text { simple total }\end{array}$} & Correlación & 0,23 & 0,23 & 0,08 & 0,04 & 0,07 & 0,04 \\
\hline & & Significancia & 0,00 & 0,00 & 0,44 & 0,68 & 0,49 & 0,70 \\
\hline & & No. obs. & 208 & 208 & 101 & 107 & 101 & 107 \\
\hline & \multirow{3}{*}{ Matemáticas } & Correlación & 0,16 & 0,16 & 0,08 & 0,04 & 0,07 & 0,04 \\
\hline & & Significancia & 0,02 & 0,03 & 0,43 & 0,65 & 0,48 & 0,65 \\
\hline & & No. obs. & 208 & 208 & 101 & 107 & 101 & 107 \\
\hline & \multirow{3}{*}{ Lenguaje } & Correlación & 0,16 & 0,16 & 0,20 & 0,17 & 0,17 & 0,15 \\
\hline & & Significancia & 0,03 & 0,02 & 0,05 & 0,08 & 0,09 & 0,12 \\
\hline & & No. obs. & 208 & 208 & 101 & 107 & 101 & 107 \\
\hline
\end{tabular}

\subsubsection{Relación con el mercado laboral}

Otro factor asociado a la formación de economistas en las instituciones de educación superior en
Colombia, especialmente los de últimos semestres, tiene que ver con la relación de éstos con el mercado laboral (Ahumada \& Butler, 2009). Respecto a la inserción al mercado laboral, la mayor proporción de

31 Fuente: Icfes (2008), Instituciones de Educación Superior. Cálculos de los Autores. 
estudiantes (el 63,65\%) de la muestra total no se encontraban laborando, mientras que el $36,35 \%$ se encontraban vinculados con ninguna actividad laboral. De la proporción de estudiantes ocupados el 63,18\% ( $21,67 \%$ de la muestra total) desempeñaron actividades relacionadas con los conocimientos adquiridos en el transcurso de su carrera (Ilustración 9). Con respecto al tipo de vinculación laboral que presentaron los estudiantes que se encontraban laborando, el 51,17\% tenían un contrato a término indefinido y el $16,76 \%$ a término fijo; lo cual sugiere la existencia de cierta estabilidad laboral para la mayoría de los estudiantes que estaban trabajando en el 2007.

El porcentaje de ocupación laboral de los estudiantes de economía en Colombia es cercano al de los estudiantes bolivianos que laboraban en el 2007, siendo entre el $40 \%$ y el $50 \%$ de la población estudiantil (Espinoza, Machicado \& Makhlouf, 2009).

Ilustración 9. Actividad laboral y relación con los conocimientos adquiridos. Muestra nacional -2007. ${ }^{32}$

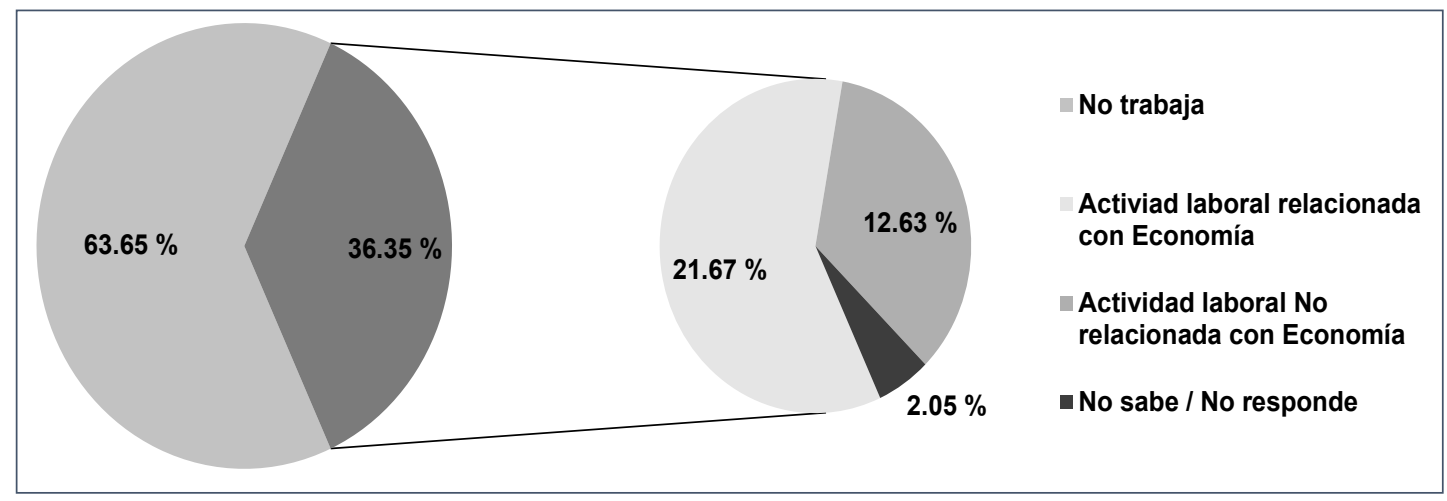

Adicionalmente, la ocupación de los estudiantes según las horas trabajadas se distribuye en tiempo completo $(63,51 \%)$, medio tiempo $(15,38 \%)$ y por horas $(13,60 \%)$. Asimismo, son empleados de empresa particular $(74,01 \%)$, empleados del gobierno $(9,75 \%)$, trabajadores por cuenta propia $(6,50 \%)$, patrones o empleadores $(2,89 \%)$ y trabajadores familiares sin remuneración (1,99\%).

Las principales actividades económicas en las que se desempeñan los estudiantes que laboran son Comercio e Intermediación financiera (Ilustración 10). Estas mismas son las principales áreas de proyección de los estudiantes, o donde desean desempeñarse una vez terminada su carrera, sin embargo se diferencian en que a pesar de que la mayor área de desempeño es comerció (28,54\%), es Intermediación financiera la mayor área de proyección (30,25\%).

\subsubsection{Factores Motivacionales}

Los aspectos motivacionales y del cumplimiento de las expectativas de los estudiantes de economía con respecto a su carrera, se encuentran relacionados con el éxito académico (Arnord \& Straten, 2012). De la totalidad de los estudiantes encuestados que respondieron si habían cumplido sus expectativas y además si querían estudiar economía, se identifica que el $82,39 \%$ de los estudiantes de los programas de economía iniciaron sus estudios porque realmente querían estudiar dicha carrera; frente al $63 \%$ correspondiente a los estudiantes de posgrado de Estados Unidos (Colander \& Klamer, 1987). Así mismo, de la muestra nacional el 79,61\% de los estudiantes consideraban que habían cumplido sus expectativas con respecto a la misma.

32 Fuente: Cálculos de los autores. 
Ilustración 10. Actividades económicas desempeñadas y de proyección según clasificación CIIU 3.1 - distribución porcentual|33

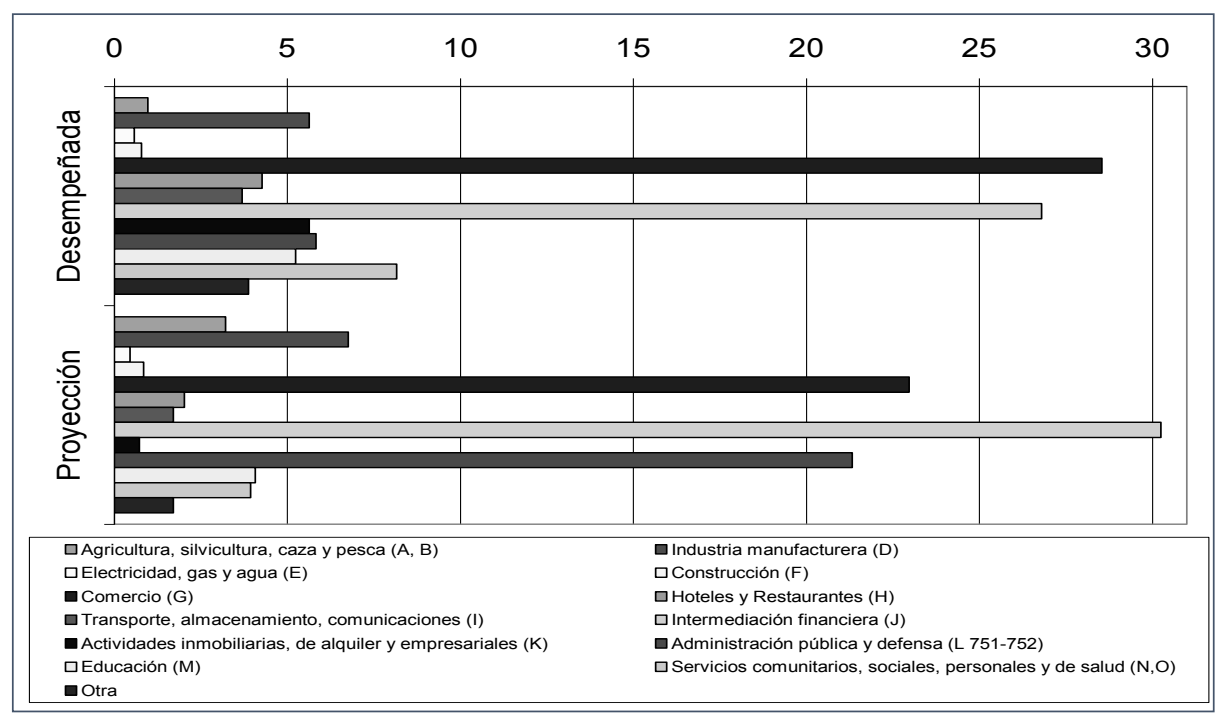

Tabla 16. Tabla de Contingencia de las preferencias y expectativas de los estudiantes por la carrera -2007. ${ }^{34}$

\begin{tabular}{|c|c|c|c|c|}
\hline \multirow{2}{*}{ ¿Queda estudiar Economía? } & \multicolumn{3}{|c|}{ ¿Ha cumplido sus expectativas? } \\
\cline { 3 - 5 } & Frecuencia & 1,066 & 235 & 1,301 \\
\hline Si & No & Total \\
\hline \multirow{2}{*}{} & Porcentaje fila & 81,94 & 18,06 & 100,00 \\
\hline & Porcentaje columna & 84,81 & 72,98 & 82,39 \\
\hline No & Porcentaje celda & 67,51 & 14,88 & 82,39 \\
\hline \multirow{2}{*}{} & Frecuencia & 191 & 87 & 278 \\
\hline & Porcentaje fila & 15,19 & 27,02 & 17,61 \\
\hline \multirow{2}{*}{ Total } & Porcentaje columna & 68,71 & 31,29 & 100,00 \\
\hline & Porcentaje celda & 12,10 & 5,51 & 17,61 \\
\hline & Frecuencia & 1,257 & 322 & 1,579 \\
\hline & Porcentaje fila & 79,61 & 20,39 & 100,00 \\
\hline & Porcentaje columna & 100,00 & 100,00 & 100,00 \\
\hline & Porcentaje celda & 79,61 & 20,39 & 100,00 \\
\hline
\end{tabular}

Existe una alta correlación entre los aspectos motivacionales, pues el $67,51 \%$ de los estudiantes encuestados querían estudiar economía y habían cumplido sus expectativas (Tabla 16). De acuerdo con la evidencia internacional el 70\% de los estudiantes mexicanos mencionaron que de empezar nuevamente sus estudios universitarios volverían a elegir economía. La proporción de estudiantes con estas misma opinión en Chile fue de 65,2\% (Ahumada \& Butler, 2009; Espinoza, Machicado \& Makholuf, 2009).

Las principales razones que mencionaron los estudiantes de economía en Colombia para el cumplimiento de sus expectativas se presentan en la Ilustración 12, entre estos se encuentran la aplicabilidad instrumental y la preferencia por su carrera, como los principales factores. En el Anexo 2 se específica en detalle en detalle la clasificación elaborada para esta variable.

\section{Perfil diferenciado de los estudiantes}

Con el fin de conocer si existen diferencias socioeconómicas de los estudiantes según ciertas características de la institución de educación superior donde adelantan estudios, se realizó un perfil diferenciado

33 Fuente: Cálculos de los autores.

34 Fuente: Cálculos de los autores. 
de los estudiantes. Esta información es útil para las instituciones debido a que permite saber a qué tipo de población se está atendiendo y plantear políticas que se adecuen a sus condiciones.

Ilustración 11. Razones del cumplimiento de expectativas de los estudiantes $-2007^{35}$

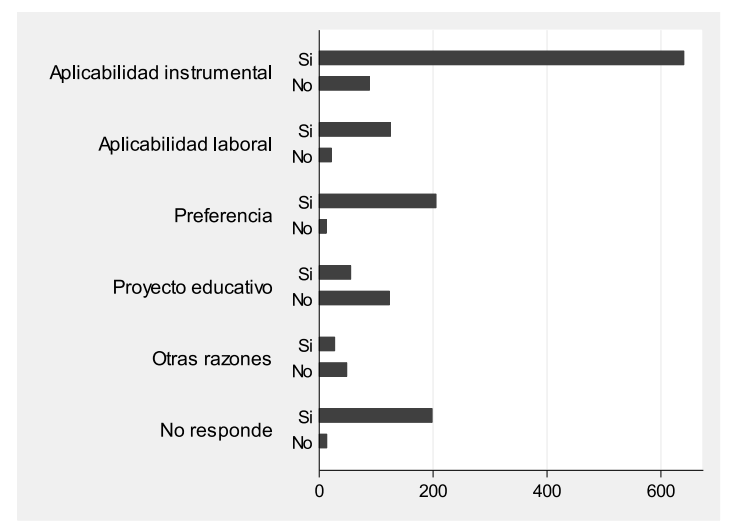

\subsection{De acuerdo al origen de la institución (publico/ privado)}

Existe un mayor número de estudiantes mujeres que hombres, además hay un mayor porcentaje de mujeres en las instituciones públicas $(58,57 \%)$ frente a las privadas $(56,27 \%)$. Esto resultados sugieren que para el caso de los estudiantes de economía en Colombia no existe un diferencia marcada en cuanto su sexo.

En el caso de la edad de los estudiantes de últimos semestres (octavo en adelante), en ambos tipos de institución la mayor concentración es en el rango de 21 a 25 años (77,72\% públicas, 66,63\% privadas); sin embargo, hay una mayor participación de estudiantes mayores de 25 años en las instituciones privadas $(20,87 \%)$ que en las públicas $(31,48 \%)$.

En referencia al estrato, las instituciones públicas atienden en mayor proporción a los estudiantes de estratos 1 y 2 que las privadas (42,79\% y 14,13\%, respectivamente) y en menor cuantía a los demás estratos. Estos resultados son acordes a lo encontrado por Ramírez (2007) en donde los estudiantes recién graduados de la educación media tienden a seleccionar la institución de educación superior de acuerdo a su estrato socioeconómico.

Por otro lado, no existieron diferencias significativas en cuanto al estado conyugal, si la persona era cabeza de familia o no y en el número de hijos. En comparación a los estudiantes de instituciones públicas, los estudiantes privados mostraron una mayor proporción de hogares para cada uno de los rangos entre uno y cuatro personas (64,59\% frente a $49,07 \%$ ); y una menor proporción de personas aportantes al ingreso del hogar para los rangos entre una y dos personas $(78,66 \%$ frente a 90,76), siendo mayor la proporción de una sola persona aportante para los públicos $(48,04 \%$,$) y de dos para los privados$ $(48,99 \%)$.

Asimismo, se calculó el grado de dependencia económica (número de aportantes al ingreso del hogar sobre el número de personas que componen el hogar), encontrándose que es mayor en el caso de las instituciones privadas que en las públicas $(0,53$ y 0,38 , respectivamente).

También se observó una mayor participación de la educación profesional y posgrados del padre en las instituciones privadas que en las públicas $(44,22 \%$ y $28,00 \%$, respectivamente), diferencia no tan marcada para el caso de la madre $(36,52 \%$ y $21,66 \%$, respectivamente).

En cuanto a sus estudios secundarios, los estudiantes de instituciones públicas estudiaron en mayor porcentaje en un colegio de carácter público $(60,77 \%)$, similar a lo que ocurre en el caso del privado en colegio privado (70,03\%). En ambos casos la mayoría estudió en un colegio de calendario A; siendo mayor la proporción de estudios secundarios normalistas y técnicos en los públicos que en los privados $(24,38 \%$ versus $15,20 \%)$.

Comparando este resultado con México, Ahumada $\&$ Butler (2009) hallaron que entre un 2 y $5 \%$ de los estudiantes que accedieron a los programas de economía cursaron su educación básica en institu-

35 Fuente: Cálculos de los autores. 
ciones públicas. Por el contrario, en las universidades públicas la mayor parte de la población estudiantil provino de escuelas de educación media superior de carácter público.

En promedio los puntajes del Icfes de la muestra nacional no presentan diferencias significativas con respecto al carácter privado o público de las instituciones de educación superior en las que estaban estudiando en el 2007; sin embargo, se observó que hay una mayor concentración sobre la media en los públicos que en los privados (Ilustración 12).

Ilustración 12. Puntaje del lcfes alcanzado según origen de la institución ${ }^{36}$

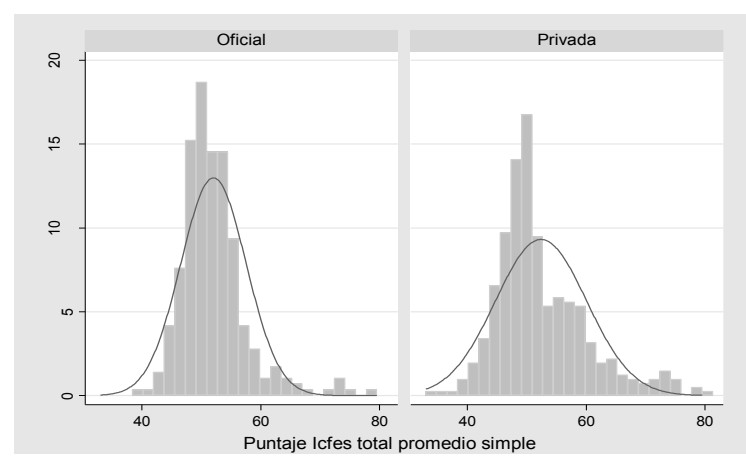

Sobre la relación entre los estudiantes y el mercado laboral, se encuentra que la mayoría de estudiantes públicos y privados no trabajan $(78,78 \%$ y $53,62 \%$, respectivamente). De los estudiantes que trabajaban, los públicos se desempeñaban en su mayoría en el comercio $(40 \%)$ y los privados en intermediación financiera $(31,39 \%)$. En el caso mexicano los estudiantes de instituciones públicas que se encontraban laborando se ocuparon en mayor proporción en el sector financiero, seguros e inmuebles; mientras que los que procedieron de instituciones privadas se emplearon en su mayoría en el sector manufacturero (Ahumada \& Butler, 2009).

De igual manera, los estudiantes de instituciones públicas se proyectaban en actividades de intermediación financiera $(28,50 \%)$ y administración públi- ca y defensa $(26,71 \%)$, mientras que los privados en intermediación financiera $(31,43 \%)$ y comercio $(27,25 \%)$.

Adicionalmente, existió una mayor estabilidad en el trabajo para los estudiantes de instituciones privadas (contratos a término fijo e indefinido) con un $36,70 \%$ frente a un $23,08 \%$ de las públicas. Consecuentemente, se encontró una menor estabilidad laboral para los estudiantes de las públicas (contratos temporales-outsourcing y prestación de servicios) con un $35,39 \%$ frente al $16,47 \%$ de los estudiantes de las privadas. En otro sentido, la mayoría de estudiantes privados trabajaron tiempo completo $(71,26 \%)$ frente al medio tiempo y por horas que trabajaron los estudiantes públicos $(48,85 \%)$.

Finalmente, los estudiantes públicos se financiaron básicamente con recursos familiares $(74,81 \%)$, y los privados con recursos familiares $(47,24 \%)$ y crédito $(24,45 \%)$.

\subsection{De acuerdo a la jornada}

En Colombia se ofrece la jornada diurna y nocturna para estudios de pregrado en economía, sin embargo con la implementación del sistema de créditos en las instituciones de educación superior se tiende a manejar una jornada única.

En la relación entre el sexo de los estudiantes y la jornada de estudio, las diferencias de representación de hombres y mujeres de acuerdo a la jornada no fueron relevantes. Además, se dio una mayor proporción de estudiantes diurnos que nocturnos en el intervalo de 21 a 25 (80,04\% para los primeros frente a $44,83 \%$ de los segundos), pues en los programas nocturnos hay una mayor proporción de estudiantes en edades de mayor rango $(53,64 \%)$.

En cuanto al nivel máximo de educación logrado por los padres, la mayoría alcanzaron hasta la educación secundaria (para el padre $28,03 \%$ de los diurnos y $33,18 \%$ de los nocturnos; para la madre $33,98 \%$ de los diurnos y $41,31 \%$ de los nocturnos); pero poseen

36 Fuente: Icfes (2008). Cálculos de los Autores. 
una mayor educación superior los padres de los estudiantes diurnos que de los nocturnos $(32,89 \%$ y $16,93 \%$ para el padre, $24,54 \%$ y $11,96 \%$ para la madre, respectivamente).

Igualmente, la distribución según estrato se encuentra concentrada en los estratos 2 a 4 para ambas jornadas $(81,08 \%$ para diurnos y $94,76 \%$ para nocturnos), pero principalmente en el estrato 3 para los diurnos y nocturnos $(38,81 \%$ y $62,41 \%$, respectivamente).

Analizando las responsabilidades personales de los estudiantes, se determina que en los nocturnos hay más casados/en unión libre (16,89\% frente a $4,09 \%)$, más estudiantes cabezas de familia $(20,18 \%$ frente a $2,18 \%$ ), y una mayor proporción de padres $(20,39 \%$, frente a $7,40 \%)$ además el grado de dependencia económica es superior $(0,62$ frente a 0,41$)$.

Por otro lado, el tiempo cesante entre la terminación de estudios secundarios y comienzo de estudios en economía es mayor para los nocturnos $(60,10 \%$ duran más de dos años) que para los diurnos $(60,54 \%$ duran hasta un año).

Estudiando los puntajes promedio del Icfes, son en promedio más elevados los de los estudiantes nocturnos $(54,09)$ que los de los diurnos $(51,48)$, pero con una mayor dispersión en los primeros (Ilustración 13).

En cuanto a la relación entre los estudiantes y el mercado laboral, el 82,21\% de los nocturnos trabaja frente al 17,50\% de los diurnos. Igualmente, su mayor participación se da en actividades de intermediación financiera $(34,34 \%$ nocturnos y $12,64 \%$ diurnos) y comercio $(25,60 \%$ nocturnos y $35,06 \%$ diurnos).

Además, hay una mayor estabilidad en los nocturnos que en los diurnos en cuanto al tipo de contrato que poseen $(80,17 \%$ y $44,39 \%$, respectivamente). Asimismo, la mayoría de los estudiantes nocturnos tienen un trabajo de tiempo completo $(84,17 \%)$ y los diurnos de medio tiempo y por horas $(63,50 \%)$.
Ilustración 13. Puntaje del Icfes alcanzado según origen de la institución ${ }^{37}$

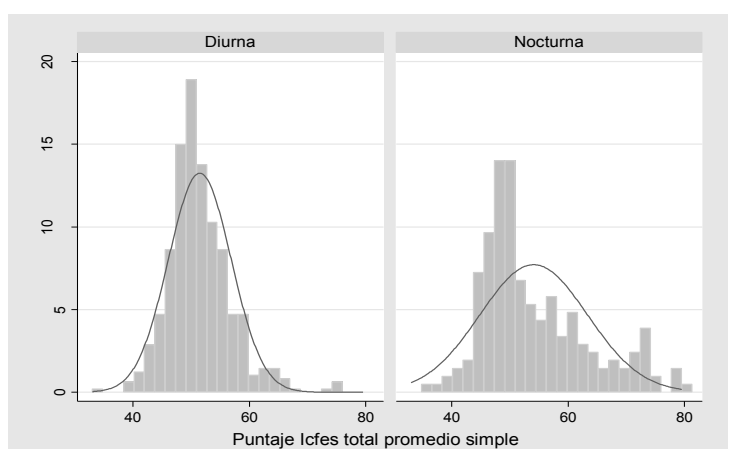

En referencia al tipo de financiación de la mayoría de los estudios de economía, los nocturnos se financian igualmente con crédito, recursos familiares y propios ( $28,48 \%$ cada una) frente a un $70,17 \%$ de recursos familiares de los diurnos.

Por último, los estudiantes nocturnos han tenido que aplazar en mayor porcentaje sus estudios de economía (29,15\% frente $10,95 \%$ de los diurnos), debido principalmente a motivos económicos $(44,53 \%)$ y laborales $(25 \%)$ en el caso de los estudiantes nocturnos, y a motivos económicos $(24,19 \%)$ y familiares $(16,94 \%)$ en el caso de los estudiantes diurnos.

\section{Comentarios finales}

Este documento caracteriza los estudiantes y las instituciones que forman economistas en Colombia. Para empezar, se enuncian los orígenes de la formación de economistas en Colombia, lo cual se dio desde híbridos con programas de Derecho a partir de 1931. Así, se analiza la evolución del número de inscritos, estudiantes matriculados y graduados, encontrándose caídas en el número de economistas graduados para los periodos 1988-1993 y 2002-2007, posiblemente debido a que han desplazado su demanda hacia otras carreras.

También se realizó una caracterización institucional de los recursos académicos y físicos de 60 instituciones de educación superior que ofrecían el programa de economía en Colombia y que presentaron estu-

37 Fuente: Icfes (2008). Cálculos de los Autores. 
diantes al Ecaes 2007, representando el 90\% de los programas que contaron con registro calificado en ese año.

En este sentido se encontró que los planes de estudio tenían en promedio 73 créditos para el componente básico y 44 para el profesional; y se componían de entre 41 y 61 asignaturas que en promedio se traducen entre 144 y 190 créditos; igualmente no se evidenció el componente de énfasis claramente, así como diferencias entre el número de asignaturas y créditos entre programas acreditados y no acreditados.

En cuanto a los recursos académicos, las instituciones acreditadas tenían un promedio mayor de monitores, más recursos bibliográficos y físicos, así como ofrecían más programas de maestría. En adición, se encontró que en general los procesos de investigación dentro de los programas se encuentran aún incipientes, debido a que de los programas que registraron grupos de investigación $(77,08 \%)$ sólo el $43,75 \%$ de estos se encontraban reconocidos por Colciencias, así como el 51,11\% de las instituciones no tenían revistas adscritas al Programa y sólo el $24,44 \%$ de las revistas se encontraban indexadas, por lo que los procesos de divulgación y calidad de los productos de investigación podrían verse disminuidos.

Por otro lado, se realizó una caracterización del estudiante de economía del 2007 en Colombia a partir de información, en su mayoría, sobre estudiantes de últimos semestres. De esta manera los estudiantes de economía son en mayor proporción mujeres, con 24 años de edad en promedio, de estratos 2,3 y 4 , cuyos padres alcanzaron en mayor proporción niveles educativos de posgrado y secundaria y con ingresos por hogar entre 2 y 3 salarios mínimos. En cuanto a su educación secundaria, estudiaron en mayor proporción en colegios privados $(56,51 \%)$ y en calendario $\mathrm{A}(85,06 \%)$.

Además, sus principales fuentes de financiación fueron recursos familiares $(58,33 \%)$ y crédito $(17,10 \%)$, dedicaron en promedio 87 minutos a la semana al estudio independiente por hora de clase, así como consultaron en promedio tres veces por semana recursos bibliográficos para sus actividades académicas.
También se evidenció que el 19,32\% ya tenían algún título, el 10,89\% se encontraban realizando otros estudios, el 29,37\% habían empezado otra carrera y no la habían terminado, y el 5,96\% había empezado estudios de economía en otra institución.

En cuanto a su desempeño en los Ecaes, se encontraron altamente correlacionados el desempeño entre los componentes de Microeconomía y Macroeconomía $(0,69)$; en este aspecto se evidencia que son mayores los puntajes Ecaes obtenidos por las instituciones públicas, las diurnas y las acreditadas.

Por otro lado, el $36,35 \%$ se encontraban laborando principalmente en las actividades económicas de Comercio e Intermediación Financiera. De los que laboraron el 63,18\% estaban realizando actividades relacionadas con la carrera.

Cabe resaltar que el $82,39 \%$ de los estudiantes querían estudiar esta carrera y el 79,61\% habían cumplido sus expectativas, principalmente porque ésta tiene aplicabilidad instrumental y por sus propias preferencias.

En cuanto a la existencia de perfiles diferenciados de acuerdo a tipificaciones de las instituciones, se encuentra que las instituciones públicas atendieron más estudiantes de los estratos 1 y 2 , así como sus estudiantes provenían en mayor proporción de colegios públicos y con estudios secundarios normalistas y técnicos. En contraste, los estudiantes de instituciones privadas provinieron en mayor proporción de colegios privados, predominó el nivel educativo de profesional y posgrado de sus padres, recurrieron más a créditos para la financiación de sus estudios y los que trabajaron tenían mayor estabilidad laboral.

De igual manera, se encontró que los estudiantes de la jornada nocturna en general, eran mayores de 25 años, tuvieron menor proporción de padres con educación superior y poseen mayores responsabilidades personales. Así, existían más estudiantes casados/unión libre, cabezas de familia, con hijos, y con mayor grado de dependencia económica, estuvieron más tiempo cesantes entre sus estudios secundarios y sus estudios de economía, aplazaron más por motivos económicos, y una mayor proporción trabajó 
tiempo completo en actividades de intermediación financiera y comercio.

Esta caracterización institucional y del perfil estudiantil podría ser utilizada para el mejoramiento continuo en la formación del economista en Colombia, con el fin de que las fortalezas desarrolladas y potenciadas en las aulas se ajusten a las necesidades de los estudiantes y de su entorno.

\section{Referencias}

Ahumada, I. \& Butler, F. (2009). La enseñanza de la economía en México. Documento de trabajo No. 672, Banco Interamericano de Desarrollo (BID).

Arnold, I. \& Straten, J. (2012). "Motivation and math skills as determinants of first-year performance in economics". En: The Journal of Economic Education, 43(1): 33-47.

Becker, W. \& Watts, M. (2001). "Teaching methods in U. S. undergraduate economics courses". En: The Journal of Economic Education, 32(3): 269-279.

Borjas, G. \& Acosta, O. (2000). Education reform in Colombia. Documentos de trabajo No. 19/2000, Fedesarrollo.

Borman, G. \& Kimball, S. (2005). "Teacher quality and educational equality: Do teachers with higher standards based evaluation ratings close student achievement gaps?". En: The Elementary School Journal, 106(1): 3-20.

Colander, D. (2003). The aging of an economist. Middlebury College Working Paper Series 0304, Middlebury College, Department of Economics.

Colander, D. \& Nopo, H. (2011). "Educating Latin American economists". En: International Review of Economics Education (IREE), 10(1): 54-69.

Colander, D. \& Klamer, A. (1987). "The making of an economist". En: The Journal of Economic Perspectives, 1(2): 95-111.

Consejo Nacional Profesional de Economía (recuperado el 1 de Agosto de 2008, de http://www.conalpe.gov.co). Cálculos de los autores.

Croninger, R., Rice, J., Rathbun, A. \& Nishio, M. (2007). "Teacher qualifications and early learning: Effects of certification, degree, and experience on first-grade student achievement". En: Economics of Education Review, 26(3): 312-324.

Decreto 2890 (1991, Diciembre 30). Diario Oficial, 42250, Diciembre 30, 1991.

Eskew, R. \& Faley, R. (1988). "Some determinants of student performance in the first college-level financial accounting course". En: The Accounting Review, 63(1): 137-147.

Espinoza, L., Machicado, C. \& Makhlouf, K. (2009). La enseñanza de economía en Bolivia y Chile. Documento de trabajo No. 673, Banco Interamericano de Desarrollo (BID).

Figlio, D. (1999). "Functional form and the estimated effects of school resources". En: Economics of Education Review, 18(2): $241-252$.

Figlio, D. \& Kenny, L. (2007). "Individual teacher incentives and student performance". En: Journal of Public Economics, 91(56): 901-914.
Gucht, F. \& Westerheijden (1993). Quality management and quality assurance in European higher education: Methods and mecanisms. Education Training Youth Studies No. 1 Commission of the European communities.

Instituto Colombiano para la Evaluación de la Educación - ICFES (recuperado el 1 de Agosto de 2008, de http://www.icfes.gov. co/investigacion/acceso-a-bases-de-datos)

Jepsen, C. (2005). Teacher characteristics and student achievement: evidence from teacher surveys. En: Journal of Urban Economics, 57(2): 302-319.

Kalmanovitz, S. (1993). "Notas para una historia de las teorías económicas en Colombia". En: Quevedo, E., Vasco, E., Obregón, D. \& Orozco, L. (Ed.). Historia Social de la Ciencia en Colombia, V. IX. Bogotá D.C: Instituto Colombiano para el Desarrollo de la Ciencia y la Tecnología Francisco José de Caldas.

Kolpin, V. \& Singell, L. (1996). "The gender composition and scholarly performance of economics departments: A test for employment discrimination". En: Industrial and Labor Relations Review, 49(3): 408-423.

Larroulet, C. \& Domper, M. (2006). La enseñanza de la economía y administración en las instituciones de educación superior. Documento de Trabajo No. 169, Instituto Libertad y Desarrollo.

Ley 37 (1990, Octubre 26). Diario Oficial, 39568, Octubre 26, 1990.

Ley 41 (1969, Diciembre 31). Diario Oficial, 32980, Enero 29, 1970.

Lora, E. \& Nopo, H. (2009). "La formación de los economistas en América Latina”. En: Revista de análisis económico, 24: 65-93.

Meisel, A. \& Pérez, G. (2005). ¿El poder a través del saber? un análisis de los exámenes de calidad de la educación superior (ECAES) de economía en Colombia realizados en el 2004. Documento Webpondo.

Montenegro, A. (2005). Los ECAES de economía. Documentos de economía No. 003164, Universidad Javeriana.

Ortiz, C. (2005). "Sobre los ECAES de Economía de 2004". En: Revista Sociedad y Economía, 8: 197-205.

Ramírez, A. (2007). Modelos de elección discreta: una aplicación a la demanda por cupos universitarios en Colombia. Documento de trabajo, Universidad EAFIT.

Resolución 2774 (2003, Noviembre 13).Diario Oficial, 45388.

Rhenals, R. (2003). De la perplejidad a la explicación: crónicas de la economía, su enseñanza y aplicación. Documento de trabajo, Universidad de Antioquia.

Rozenwurcel, G., Bezchinsky, G. \& Rodríguez, M. (2009). La enseñanza de economía en Argentina. Documento de trabajo No. 671, Banco Interamericano de Desarrollo (BID).

Sarmiento, J. \& Sandoval, L. (2008). "Análisis descriptivo de los resultados de los ECAES en economía en Colombia". En: Revista Facultad de Ciencias Económicas: Investigación y Reflexión, XVI(2): 79-104.

Silva, C. \& Sarmiento, J. (2006). "Estudiantes de economía de la UMNG, ¿Cómo son?" En: Revista Facultad de Ciencias Económicas: Investigación y Reflexión, XIV(1): 238-257.

Sistema Nacional de Información de Educación Superior -SNIES Ministerio de Educación Nacional (recuperado el 1 de Agosto de 2008, de http://bi.mineducacion.gov.co:8380/eportal/web/ snies1/poblacion-estudiantil).

Universidad de Antioquia (2003). Alternativas a la tiza y el tablero. Métodos de enseñanza en economía: un análisis comparativo. Documento de trabajo, Universidad de Antioquia. 
Valens, M. (2007). "Calidad de la educación superior en Colombia: Un análisis multinivel con base en el ECAES de economía 2004". En: Revista Sociedad y Economía, 13: 132-154.

Virgüez, A., Silva, C. \& Sarmiento, J. (2013). Evolución de la calidad de los programas de economía en Colombia. Un análisis desde los resultados ECAES de 2004 y 2008. Trabajo no publicado. Bogotá: Universidad Militar Nueva Granada.
Walstad, W. \& Rebeck, K. (2001). "Teacher and student economic understanding in transition economics". En: Journal of Economic Education, 32: 58-67.

Woessmann, L. (2003). Educational production in East Asia: The impact of family background and schooling policies on student performance. Kiel Working Papers 1152, Kiel Institute for the World Economy. 


\section{Anexos}

Tabla 17. Muestra de instituciones y/o estudiantes por característica - $2007^{39}$

\begin{tabular}{|c|c|c|c|c|}
\hline & $\begin{array}{l}\text { Número de Insti- } \\
\text { tuciones }\end{array}$ & $\begin{array}{l}\text { Número de } \\
\text { estudiantes }\end{array}$ & $\begin{array}{l}\text { Representatividad } \\
\text { total programas de } \\
\text { economía (2007) }\end{array}$ & Fuente \\
\hline \multicolumn{5}{|l|}{ Carácterísticas programa } \\
\hline \multicolumn{5}{|l|}{ Caracterización institucional } \\
\hline Ubicación geográfica & 60 & N/A & $90 \%$ & Ministerio de Educación Nacional - MEN \\
\hline Valor matrícula & 52 & $\mathrm{~N} / \mathrm{A}$ & $78 \%$ & $\begin{array}{l}\text { Fuente directa programas de economía, } \\
\text { otras fuentes }\end{array}$ \\
\hline Jornada & 37 & N/A & $55 \%$ & Fuente directa programas de Economía \\
\hline Condicion de acreditación & 60 & N/A & $90 \%$ & $\begin{array}{l}\text { Fuente directa programas de Economía } \\
\text { y CNA }\end{array}$ \\
\hline Origen de la institución & 60 & N/A & $90 \%$ & Ministerio de Educación Nacional - MEN \\
\hline \multicolumn{5}{|l|}{ Recursos académicos } \\
\hline Plan de estudios & 53 & N/A & $79 \%$ & $\begin{array}{l}\text { Fuente directa programas de Economía. } \\
\text { Otras fuentes }\end{array}$ \\
\hline Plan de estudios por créditos & 35 & $\mathrm{~N} / \mathrm{A}$ & $52 \%$ & Fuente directa programas de Economía \\
\hline Duración carrera & 49 & $\mathrm{~N} / \mathrm{A}$ & $73 \%$ & Fuente directa programas de Economía \\
\hline Número de monitores & 27 & $\mathrm{~N} / \mathrm{A}$ & $40 \%$ & Fuente directa programas de Economía \\
\hline Programas de postgrado & 49 & N/A & $73 \%$ & $\begin{array}{l}\text { Fuente directa programas de Economía. } \\
\text { Otras fuentes }\end{array}$ \\
\hline Grupos de investigación & 48 & N/A & $72 \%$ & $\begin{array}{l}\text { Fuente directa programas de Economía. } \\
\text { Otras fuentes }\end{array}$ \\
\hline Revistas adscritas & 45 & $\mathrm{~N} / \mathrm{A}$ & $67 \%$ & $\begin{array}{l}\text { Fuente directa programas de Economía. } \\
\text { Otras fuentes }\end{array}$ \\
\hline Aspectos sobre el profesorado & 15 & $\mathrm{~N} / \mathrm{A}$ & $22 \%$ & Fuente directa programas de Economía \\
\hline \multicolumn{5}{|l|}{ Recursos físicos y administrativos } \\
\hline Personal administrativo programa & 26 & N/A & $39 \%$ & Fuente directa programas de Economía \\
\hline Personal administrativo institución & 17 & N/A & $25 \%$ & Fuente directa programas de Economía \\
\hline Bibliográficos (libros) & 33 & $\mathrm{~N} / \mathrm{A}$ & $49 \%$ & Fuente directa programas de Economía \\
\hline Computadores & 26 & N/A & $39 \%$ & Fuente directa programas de Economía \\
\hline \multicolumn{5}{|l|}{ Características de estudiantes } \\
\hline \multicolumn{5}{|l|}{ Características socioeconómicas } \\
\hline Sexo & 60 & 3402 & $90 \%$ & Encuesta a estudiantes e ICFES \\
\hline Edad & 60 & 3349 & $90 \%$ & Encuesta a estudiantes e ICFES \\
\hline Estrato socioeconómico & 60 & 3359 & $90 \%$ & Encuesta a estudiantes e ICFES \\
\hline Escolaridad máxima padre & 59 & 3294 & $88 \%$ & Encuesta a estudiantes e ICFES \\
\hline Escolaridad máxima madre & 59 & 3349 & $88 \%$ & Encuesta a estudiantes e ICFES \\
\hline Situación conyugal & 60 & 3396 & $90 \%$ & Encuesta a estudiantes e ICFES \\
\hline
\end{tabular}

39 Fuente: los Autores. 


\begin{tabular}{|c|c|c|c|c|}
\hline Cabeza de familia & 37 & 1619 & $55 \%$ & Encuesta a estudiantes e ICFES \\
\hline Número de hijos & 37 & 1423 & $55 \%$ & Encuesta a estudiantes e ICFES \\
\hline Número de personas en el hogar & 37 & 1609 & $55 \%$ & Encuesta a estudiantes e ICFES \\
\hline Ingreso familiar & 37 & 1558 & $55 \%$ & Encuesta a estudiantes e ICFES \\
\hline \multicolumn{5}{|l|}{ Educación secundaria } \\
\hline Tipo de colegio & 37 & 1621 & $55 \%$ & Encuesta a estudiantes e ICFES \\
\hline Calendario del colegio & 37 & 1613 & $55 \%$ & Encuesta a estudiantes \\
\hline Jornada del colegio & 37 & 1621 & $55 \%$ & Encuesta a estudiantes \\
\hline Titulo de bachiller & 60 & 3386 & $90 \%$ & Encuesta a estudiantes e ICFES \\
\hline Puntaje Icfes & 36 & 702 & $54 \%$ & ICFES \\
\hline \multicolumn{5}{|l|}{ Desenvolmiento en la carrera } \\
\hline Fuente de financiación & 37 & 1608 & $55 \%$ & Encuesta a estudiantes e ICFES \\
\hline Dedicación al estudio & 37 & 1202 & $55 \%$ & Encuesta a estudiantes \\
\hline Formación previa & 37 & 1615 & $55 \%$ & Encuesta a estudiantes \\
\hline Formación paralela & 37 & 1607 & $55 \%$ & Encuesta a estudiantes \\
\hline \multicolumn{5}{|l|}{ Resultados académicos trayectoria estudiantil } \\
\hline PGA (Total y por componentes) & 6 & 191 & $9 \%$ & Fuente directa programas de Economía \\
\hline Puntaje Ecaes (Total y por componentes) & 58 & 2224 & $87 \%$ & ICFES \\
\hline \multicolumn{5}{|l|}{ Relación con el mercado laboral } \\
\hline Inserción mercado laboral & 37 & 1560 & $55 \%$ & Encuesta a estudiantes \\
\hline Tipo de vinculación laboral & 37 & 555 & $55 \%$ & Encuesta a estudiantes \\
\hline Dediación laboral & 37 & 559 & $55 \%$ & Encuesta a estudiantes \\
\hline Actividad económica & 35 & 515 & $52 \%$ & Encuesta a estudiantes \\
\hline Expectativas desempeño actividad económica & 35 & 1424 & $52 \%$ & Encuesta a estudiantes \\
\hline \multicolumn{5}{|l|}{ Factores motivacionales } \\
\hline ¿Quería estudiar economía? & 37 & 1605 & $55 \%$ & Encuesta a estudiantes \\
\hline ¿Ha cumplido sus expectativas? & 37 & 1591 & $55 \%$ & Encuesta a estudiantes \\
\hline Razón de expectativas & 37 & 1591 & $55 \%$ & Encuesta a estudiantes \\
\hline
\end{tabular}


Tabla 18. Razones cumplimiento de expectativas ${ }^{40}$

\begin{tabular}{|c|c|}
\hline Clasificación & Explicación \\
\hline Aplicabilidad instrumental & $\begin{array}{l}\text { El estudiante asocia el instrumento a una aplicación específica, generalmente se resalta la importancia de } \\
\text { la fundamentación macroeconómica y la posibilidad que la misma les proporciona para entender, analizar } \\
\text { y trabajar en temas de importancia nacional. Se encuentra que los estudios en economía permiten la com- } \\
\text { prensión y entendimiento de los fenómenos económicos, el funcionamiento de los mercados y la situación } \\
\text { socioeconómica del país. También se considera que es aplicable a la sida diaria. Igualmente, se refiere al } \\
\text { conjunto de herramientas, conocimientos y fundamentos sólidos adquiridos en la formación en economía. } \\
\text { Por lo general no lo asocian con una aplicación específica, pero manifiestan que aumenta su capacidad de } \\
\text { análisis, de razonamiento, y amplia su visión por la diversidad de enfoques. En sus respuestas manifiestan } \\
\text { que la carrera es muy completa, y que es integral al abarcar muchos campos, conceptos y teorías. }\end{array}$ \\
\hline Aplicabilidad laboral & $\begin{array}{l}\text { Se refiere explícitamente a que el cumplimiento o no de sus expectativas está asociado con la aplicabilidad } \\
\text { en la vida laboral de los conocimientos y herramientas adquiridas. En este sentido para algunos estudiantes } \\
\text { los estudios en economía les han permitido un mejor desempeño laboral. Para otros, que no han incursionado } \\
\text { al mercado laboral, aumenta sus posibilidades laborales o consideran que les proporciona las condiciones } \\
\text { necesarias para crear su propia empresa. }\end{array}$ \\
\hline Preferencia & $\begin{array}{l}\text { Satisfacción, agrado e interés por los temas vistos y los conocimientos adquiridos en la carrera. Los estu- } \\
\text { diantes se refieren a la motivación, y aquellos que inicialmente no querían estudiar economía manifiestan que } \\
\text { el cumplimiento de las expectativas se debe a que no esperaban que fuera tan interesante o comprendiera } \\
\text { tanto conocimiento. }\end{array}$ \\
\hline Proyecto educativo & $\begin{array}{l}\text { Manifiesta satisfacción o insatisfacción por aspectos como: } \\
\text { 1. La composición de los planes de estudios: las materias énfasis ofrecidos. } \\
\text { 2. La enseñanza: calidad de los profesores. } \\
\text { 3. La institución: su calidad, planta fisica y el criterio para la selección de profesores }\end{array}$ \\
\hline Otras razones & $\begin{array}{l}\text { Las razones que se dan para el cumplimiento de las expectativas corresponden a temas como el énfasis de la } \\
\text { carrera, Ios contenidos de las asignaturas o los fundamentos teóricos pero no es claro si se deberían atribuir a } \\
\text { fallas del proyecto educativo, y se considera prudente que sean clasificadas como apreciaciones generales de } \\
\text { la carrera de economía. En las respuestas los estudiantes manifiestan cierto tipo de inconformismo positivo } \\
\text { cuando manifiestan que sus expectativas nunca serán completamente satisfechas y que siempre habrá algo } \\
\text { por aprender. De otra parte, algunas razones no siguen una línea definida o nos son claras y por tanto se } \\
\text { clasifican en esta categoría. }\end{array}$ \\
\hline No responde & Los estudiantes responden a la pregunta del cumplimiento de las expectativas pero no especifican la razón \\
\hline
\end{tabular}

40 Fuente: Icfes (2008). Cálculos de los Autores. 